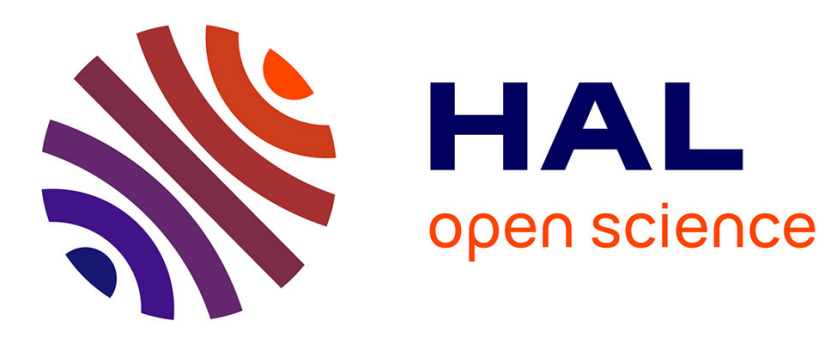

\title{
Bayesian Estimation for Optimized Structured Illumination Microscopy
}

François Orieux, Eduardo Sepulveda, Vincent Loriette, Benoit Dubertret, Jean-Christophe Olivo-Marin

\section{- To cite this version:}

François Orieux, Eduardo Sepulveda, Vincent Loriette, Benoit Dubertret, Jean-Christophe OlivoMarin. Bayesian Estimation for Optimized Structured Illumination Microscopy. IEEE Transactions on Image Processing, 2012, 10.1109/TIP.2011.2162741 • hal-01225862

\section{HAL Id: hal-01225862 \\ https://hal.science/hal-01225862}

Submitted on 10 Nov 2015

HAL is a multi-disciplinary open access archive for the deposit and dissemination of scientific research documents, whether they are published or not. The documents may come from teaching and research institutions in France or abroad, or from public or private research centers.
L'archive ouverte pluridisciplinaire HAL, est destinée au dépôt et à la diffusion de documents scientifiques de niveau recherche, publiés ou non, émanant des établissements d'enseignement et de recherche français ou étrangers, des laboratoires publics ou privés. 


\title{
Bayesian Estimation for Optimized Structured Illumination Microscopy
}

\author{
François Orieux*, Eduardo Sepulveda, Vincent Loriette, Benoît Dubertret, Jean-Christophe Olivo-Marin*
}

\begin{abstract}
Structured Illumination Microscopy is a recent imaging technique that aims at going beyond the classical optical resolution by reconstructing high resolution images from low resolution images acquired through modulation of the transfer function of the microscope. The classical implementation [1] has a number of drawbacks, like requiring a large number of images to be acquired and parameters to be set manually in an ad-hoc manner that have, until now, hampered its wide dissemination. Here, we present a new framework based on a Bayesian inverse problem formulation approach that enables the computation of one high-resolution image from a reduced number of low-resolution images and has no specific constraints on the modulation. Moreover, it permits to automatically estimate the optimal reconstruction hyper-parameters and to compute an uncertainty bound on the estimated values. We demonstrate through numerical evaluations on simulated data and examples on real microscopy data that our approach represents a decisive advance for a wider use of high-resolution microscopy through structured illumination.
\end{abstract}

Index Terms-Structured illumination microscopy, image reconstruction, microscopy, deconvolution, super-resolution, Bayesian inversion, MCMC.

\section{INTRODUCTION}

\section{A. Superresolution Microscopy}

O PTICAL microscopy is one of the major research tools of modern biology as it opens the way to study the links between cellular phenotypes and the underlying molecular mechanisms through the quantitative imaging of cell compartment dynamics [2], [3]. Classical optical microscopes are, however, limited to a lateral resolution of 200 nanometers and a 600 nanometer axial resolution imposed, by the phenomenon of diffraction of light by the microscope objective pupil, which does not allow for resolving a large class of sub-resolution biological objects.

Two active fields of research aim at advancing the capabilities of optical microscopes either by increasing the resolution or by improving the localization accuracy of individual objects [4], [5]. Examples of the latter are recent techniques like PALM [6] or STORM [7]: individual objects are excited with a high probability of being physically separated from their

Copyright (c) 2010 IEEE. Personal use of this material is permitted However, permission to use this material for any other purposes must be obtained from the IEEE by sending a request to pubs-permissions@ieee.org

F. Orieux and J.-C. Olivo-Marin are with the Quantitative Image Analysis unit, Institut Pasteur, CNRS URA 2582, Paris, France. E-mail: \{orieux,jcolivo\}@pasteur.fr.

E. Sepulveda, V. Loriette and B. Dubertret are with Laboratoire de Physique et d'Étude des Matériaux, CNRS UMR 8213, ESPCI-ParisTech, Paris, France. E-mail: \{edo,vincent.loriette,benoit.dubertret\}@espci.fr.

This project has been financed by ANR, Institut Pasteur and ESPCI. FO was funded through an ANR fellowship. neighbors and therefore, a highly accurate estimation of their position can be computed. An image is constructed by accumulating individual objects' positions. It should be stressed that strictly speaking, no fundamental resolution enhancement is achieved in this case as it is not possible to distinguish two objects closer than the classical diffraction limit had they been excited simultaneously.

In the former approaches, the idea is to modify the sample illumination process in such a way that the classical diffraction limit does not hold anymore and higher spatial frequencies can be recovered, which means a true gain in resolution. For example, confocal microscopy [8], besides enabling 3D imaging of biological samples, has improved the lateral resolution by a factor of 1.4 by using conjugated pinholes to reject out-offocus light. Similarly, two-photon microscopy [9] and more recently STED [10] have also increased the resolution by restricting the excitation volume. As powerful as they are, these techniques present the drawback of making inefficient use of photons as the ratio between the number of detected fluorescence photons to the number of excitation photons is very low. As an alternative to these scanning approaches, full field techniques like 4-pi microscopy [10] and Structured Illumination Microscopy (SIM) [1] have better photon efficiency because most of the photons are collected and used to form the image. Even if the resolution power of SIM approach is lower compared to STED, the good efficiency and the wide field illumination constitute an important counterpart.

\section{B. A signal processing interpretation of SIM}

Diffraction theory states that incoherent ${ }^{1}$ optical systems can be described by the impulse response (or point spread function - PSF) obtained from the square of the Fourier transform of the aperture [11]. The corresponding transfer function (OTF) is the auto-correlation of the aperture and is equal to zero for all frequencies beyond a cutoff frequency $f_{c}$. In this case, all the information outside this bound is completely lost. This phenomenon is illustrated in Fig. 1 where all the frequencies outside the support (in dotted black) of the transfer function are not observed.

The idea of structured illumination microscopy (SIM) [1], [12], [13] is to inject high-frequencies inside the support of the transfer function with the help of amplitude modulation before convolution. In other words, the objective of the illumination is to introduce aliasing through modulation, as illustrated in Fig. 1. and to gain information in the frequency range beyond $f_{c}$ in order to reconstruct a high resolution (HR) image.

\footnotetext{
${ }^{1}$ Coherent systems are addressed in Sec. IV-B
} 
Let us denote the original image $f(x, y) \in L_{2}$ where $(x, y) \in \mathbb{R}^{2}$ and its continuous Fourier transform $F\left(f_{x}, f_{y}\right)$ (also called spectrum). In the standard SIM setup, the illumination modulation pattern is

$$
m(x, y)=I_{0}\left(1+\alpha \cos \left(2 \pi\left(k_{x} x+k_{y} y+\phi\right)\right)\right)
$$

and takes its values in $\left[I_{0}(1-\alpha), I_{0}(1+\alpha)\right]$ (the illumination, being an intensity, is necessarily positive). The parameter $\alpha$ is the modulation depth, whose value depends on the coherent or incoherent nature of the emission light, on the aberrations induced by the observed sample and the overall quality of the optical setup. Its Fourier transform is

$$
\begin{aligned}
M\left(f_{x}, f_{y}\right)= & I_{0}\left(\delta\left(f x, f_{y}\right)+\right. \\
& \frac{\alpha}{2} \delta\left(f_{x}-k_{x}, f_{y}-k_{y}\right) e^{-2 i \pi \phi}+ \\
& \left.\frac{\alpha}{2} \delta\left(f_{x}+k_{x}, f_{y}+k_{y}\right) e^{-2 i \pi \phi}\right) .
\end{aligned}
$$

With this modulation, the main spectrum $F\left(f_{x}, f_{y}\right)$ is replicated three times and centered in $(0,0),\left(k_{x}, k_{y}\right)$ and $\left(-k_{x},-k_{y}\right)$. Let us now introduce the convolution with the PSF $h(x, y)$, corresponding to a window in the Fourier domain with the transfer function $H\left(f_{x}, f_{y}\right)$ as

$$
\begin{aligned}
G\left(f_{x}, f_{y}\right)= & I_{0} H\left(f_{x}, f_{y}\right)\left(F\left(f_{x}, f_{y}\right)+\right. \\
& \frac{\alpha}{2} F\left(f_{x}-k_{x}, f_{y}-k_{y}\right) e^{-2 i \pi \phi}+ \\
& \left.\frac{\alpha}{2} F\left(f_{x}+k_{x}, f_{y}+k_{y}\right) e^{-2 i \pi \phi}\right)
\end{aligned}
$$

where $G\left(f_{x}, f_{y}\right)$ represents the data in the Fourier domain. Since the transfer function $H$ is not replicated, the weighting coefficients are different for each term in $(3)$.

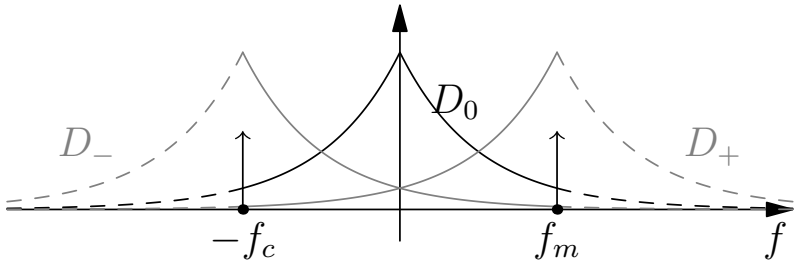

Fig. 1. Amplitude modulation of a signal. The original pattern (solid black) is centered around 0 . The replications, due to modulation (light gray), are centered about the modulation frequencies $f_{m}$ and $-f_{m}$. Outside the support of the transfer function, the frequencies are null, represented in dashed line. With the modulation, High-frequencies from $f_{c}$ to $2 f_{m}$ are inside the support through the modulation.

This concept, illustrated for one specific orientation in Fig. 2a. allows to measure frequencies present in an extended support. By repeating the same modulation, but with different orientations, a tiling can be constructed giving raise to measurements in a full ring outside the classical support of the PSF as illustrated in Fig. $2 \mathrm{~b}$.

\section{Existing approaches}

The mere fact of introducing aliasing as done in Eqn. (3) is not sufficient to obtain a HR (High Resolution) image. Indeed, with just one LR image (Low Resolution), aliased frequencies are summed up and there is no way to solve the

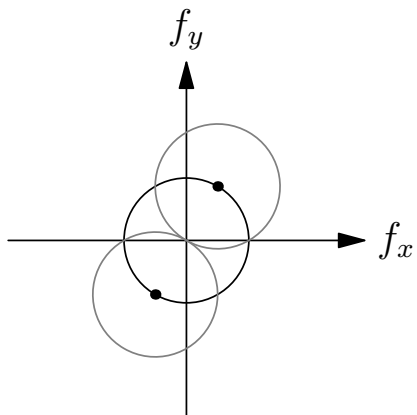

(a)

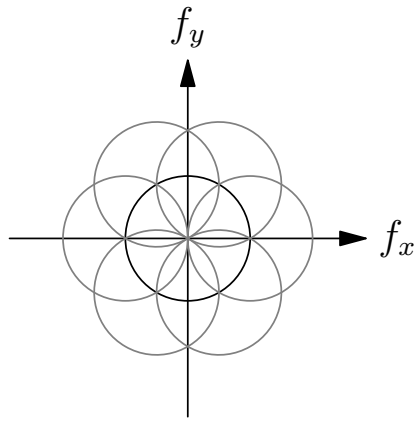

(b)
Fig. 2. Measure of high-frequency by modulation. Fig. 2a illustrates the modulation with the reproduction, in gray, of the central pattern around the two Dirac (black dot). Fig. $2 b$ illustrates the tiling for the measure of highfrequencies in a second ring.

ambiguity problem unless making use of supplementary information. This can be done either by acquiring additional LR images with different illumination patterns, or by a different modulation.

In the original method [12]-[14], each pattern centered on $(0,0),\left(k_{x}, k_{y}\right)$ and $\left(-k_{x},-k_{y}\right)$ is considered to be a different unknown. From Eqn. (3) we can write

$$
\begin{aligned}
G\left(f_{x}, f_{y}\right)= & I_{0}\left(H\left(f_{x}, f_{y}\right) F\left(f_{x}, f_{y}\right)+\right. \\
& \frac{\alpha}{2} H\left(f_{x}, f_{y}\right) F\left(f_{x}-k_{x}, f_{y}-k_{y}\right) e^{-2 i \pi \phi}+ \\
& \left.\frac{\alpha}{2} H\left(f_{x}, f_{y}\right) F\left(f_{x}+k_{x}, f_{y}+k_{y}\right) e^{-2 i \pi \phi}\right) \\
= & H\left(f_{x}, f_{y}\right)\left(F\left(f_{x}, f_{y}\right)+F_{-}\left(f_{x}, f_{y}\right)+F_{+}\left(f_{x}, f_{y}\right)\right) . \\
= & D_{0}\left(f_{x}, f_{y}\right)+D_{-}\left(f_{x}, f_{y}\right)+D_{+}\left(f_{x}, f_{y}\right) .
\end{aligned}
$$

Aliasing appears through the sum of the three components $D_{0}, D_{-}$and $D_{+}$, and in this case, the resolution of the system consists in separating the three components for each orientation. By observing that the angular term $-2 i \pi \phi$ appears only in the replication $D_{-}$and $D_{+}$, the classical solution relies on using three different patterns for each orientation, which are shifted in phases relative to one another by $2 \pi / 3$. We can, therefore, rewrite (6) as:

$$
\begin{array}{r}
G_{k}\left(f_{x}, f_{y}\right)=D_{0}\left(f_{x}, f_{y}\right)+D_{-}\left(f_{x}, f_{y}\right) e^{-2 k i \pi / 3}+ \\
D_{+}\left(f_{x}, f_{y}\right) e^{2 k i \pi / 3} .
\end{array}
$$

where $k=0,1$ or 2 . Thereafter, thanks to the choice of the $2 \pi / 3$ phase shift, the system can be solved by linear combinations between the LR images as:

$$
\begin{aligned}
D_{0} & =\frac{1}{3}\left(G_{0}+G_{1}+G_{2}\right) \\
D_{-} & =\frac{1}{3}\left(G_{0}+e^{4 i \pi / 3} G_{1}+e^{-4 i \pi / 3} G_{2}\right) \\
D_{+} & =\frac{1}{3}\left(G_{0}+e^{-4 i \pi / 3} G_{1}+e^{4 i \pi / 3} G_{2}\right) .
\end{aligned}
$$

Following this component separation for one orientation, the classical approaches by Gustafsson [1] or Mandula [13] go through a number of different processing steps:

- registration of sub-patterns, 
- correction of the weighting by transfer function,

- new combination of the sub-patterns with the new weighting factors,

- final reconstruction through Wiener filtering.

Overall, these methods suffers from several drawbacks. First, the use of the Wiener filter is problematic: to regularize the problem, it does require prior information, which usually involves at least an SNR (Signal to Noise Ration) parameter unknown in real-life problems. Effectively, the SNR parameter depends on the noise power, that can be estimated, but also on the unknown signal. Consequently, this regularization parameter is generally tuned in an $a d$-hoc way. Also, this approach requires at least three images per modulation orientation, with a total of nine LR images to reconstruct a single HR image. Finally, the existing approaches do not provide a rigorous formalism with which to derive an uncertainty bound on the estimation.

\section{Development of this paper}

In this paper, we propose a new global and coherent Bayesian framework, to compute the high-resolution (HR) image ${ }^{2}$, which is able to address all the previous drawbacks. It relies on an inverse problem formulation approach that enables the computation of one HR image from just four low-resolution images and without any constraint on the modulation pattern (like for example, the $2 \pi / 3$ phase shifting factor). Moreover, within this Bayesian framework [15], it is possible to rigorously formulate prior information about the system and to estimate jointly all the unknown regularization parameters. Finally, the Bayesian framework also permits to estimate an uncertainty bound about the reconstructed image and the associated parameters. The proposed method is more objective and requires less or no tuning by the user.

The paper is organized as follows. The proposed models and algorithms are presented in Section III. Section III discusses aspects related to the number of LR images. Section IV] is devoted to experiments and results.

\section{BAYESIAN INVERSION FOR SIM}

The reconstruction of an HR image can be seen as an inverse problem, i.e., the estimation of the original image that is at the origin of the data. There is abundant literature about this subject [16]-[19].

A powerful framework to address the inversion issue is the Bayesian approach. In this framework, all the available information about the unknowns $[f, \gamma]$ in an uncertain environment is contained in the posterior law $p(\boldsymbol{f}, \boldsymbol{\gamma} \mid \boldsymbol{g})$, where $\boldsymbol{f}$ is the variable of interest, $\gamma$ the hyper-parameters and $\boldsymbol{g}$ the data. By applying the Bayes rules, we get

$$
p(\boldsymbol{f}, \boldsymbol{\gamma} \mid \boldsymbol{g})=p(\boldsymbol{g} \mid \boldsymbol{f}, \boldsymbol{\gamma}) p(\boldsymbol{f}, \boldsymbol{\gamma}) / p(\boldsymbol{g}) .
$$

This posterior law can be obtained thanks to the product of the likelihood $p(\boldsymbol{g} \mid \boldsymbol{f}, \boldsymbol{\gamma})$, that models all the information

\footnotetext{
${ }^{2}$ The term resolution is used in the sense of spectral content here and not in the sense of sampling frequency.
}

coming from the data and their uncertainty, and the prior law of the unknowns $p(\boldsymbol{f}, \boldsymbol{\gamma})$ that models the information coming from other sources (like the classical smoothness). This is an inverse formula that allows determining the unknown causes (the image $\boldsymbol{f}$ ) from the known effects (the data $\boldsymbol{g}$ ).

\section{A. Forward model}

The forward model aims to simulate or reproduce the $L$ LR data images acquisition $\boldsymbol{g}_{l} \in \mathbb{R}^{N}$, indexed by $l$, from the original HR image $f \in \mathbb{R}^{N}$. The original image $f$ is written as a vector where the lines of the image are column stacked. As described in Sec. I. there are two main elements to be modeled: the illumination and the convolution by the optical setup.

The illumination is an amplitude modulation by an excitation light. Each pixel of the image or each element of $f$ is weighted by a cosine pattern. This operation is described by the multiplication of $\boldsymbol{f}$ with a diagonal matrix $\boldsymbol{M}_{l}$ where the diagonal elements correspond to the modulation pattern of Eq. (1). This matrix depends on the modulation parameters, different for each $l$ LR image. Since we consider the case of an ideal illumination model Eq. (1), the influence of the optical setup reduces to a change in the contrast of the grid, easily modeled by the $\alpha$ parameter described previously and whose value is fixed by the optical setup and the sample. For coherent illumination, the OTF is flat and has therefore no influence on $\alpha$. For incoherent illumination, the OTF decreases with frequency and the value of $\alpha$ is attenuated, whereby fringes become less visible. In any case, $\alpha$ must be either fixed in an ad-hoc manner or estimated from the data.

After modulation, the optical system collects the light and focuses it on a detector. The lens and the detector global response can be modeled, in the incoherent light regime, as a convolution that combines both responses. The convolution operation is written as a product between the modulated image and the matrix $\boldsymbol{H}$. This is a Toeplitz matrix and its first line corresponds to the discrete 2D PSF $H$.

These two previous operations are combined to model the full acquisition. For the acquisition of one data image we have

$$
\boldsymbol{g}_{l}=\boldsymbol{H} \boldsymbol{M}_{l} \boldsymbol{f}+\boldsymbol{n}_{l}
$$

where $\boldsymbol{n}_{l}$ is an unknown term that models the model error and the noise.

If the matrix $\boldsymbol{H}$ is block-circulant circulant-block (BCCB), it can be diagonalized in Fourier domain $\boldsymbol{F}^{\dagger} \boldsymbol{\Lambda}_{\boldsymbol{H}} \boldsymbol{F}=\boldsymbol{H}$, where $\boldsymbol{F}$ is the unitary Fourier transform and $\boldsymbol{\Lambda}_{\boldsymbol{H}}$ a diagonal matrix. This is the discrete formulation of the duality of the convolution in direct and Fourier domain. In this case, the model becomes

$$
\boldsymbol{g}_{l}=\boldsymbol{F}^{\dagger} \boldsymbol{\Lambda}_{\boldsymbol{H}} \boldsymbol{F} \boldsymbol{M}_{l} \boldsymbol{f}+\boldsymbol{n}_{l} .
$$

By collecting all the $L$ LR images in one vector $\boldsymbol{g}=$ $\left[\boldsymbol{g}_{1}, \boldsymbol{g}_{2}, \ldots \boldsymbol{g}_{L}\right]$ (same thing for $\boldsymbol{n}$ ), the forward model can be written as

$$
g=\bar{H} M R f+n
$$


where $\boldsymbol{R}=\left[\boldsymbol{I}^{t} \boldsymbol{I}^{t} \ldots\right]^{t}$ is a replication matrix, $\boldsymbol{M}$ a block diagonal matrix with $\boldsymbol{M}_{l}$ in each block and $\overline{\boldsymbol{H}}$ a block diagonal matrix with $\boldsymbol{H}$ in each block.

An important issue about this forward model is that the matrix $M$ can model any kind of amplitude modulation, allowing a very flexible framework to design efficient illumination. In particular, this modeling allows us to reduce the number of necessary images as described in the next section and illustrated in Sec. IV]

\section{B. Likelihood}

The noise term $\boldsymbol{n}$ is known up to statistical information. The choice of the noise law depends mainly on the application and most cases in image processing use either Gaussian or Poisson distributions. In the case of position dependent noise, Poisson or non-homogeneous Gaussian distributions can be used with the difficulty that in the former case the possible estimators are no longer linear wrt. data, while in the latter the covariance matrix needs to be estimated through quite cumbersome procedures.

In applications where a high number of photons are collected, some simplifications occur. First, the Poisson law tends towards a Gaussian. Then, the noise introduced by readout electronics can be described accurately by a Gaussian statistics. Finally, even if the standard deviation depends on the position, a Variance Stabilizing Transform like in [20] can be used to stabilize it. For these reasons, our proposed method uses an iid Gaussian law

$$
\boldsymbol{n}_{l} \sim \mathcal{N}\left(\mathbf{0}, \gamma_{\boldsymbol{n}_{l}}^{-1} \boldsymbol{I}\right)
$$

where $\gamma_{\boldsymbol{n}_{l}}$ is the unknown inverse variance for this LR image. Consequently, the data law when the HR image is known, or the likelihood, is also Gaussian:

$$
\begin{aligned}
p\left(\boldsymbol{g}_{l} \mid \boldsymbol{f}, \gamma_{\boldsymbol{n}_{l}}\right)= & (2 \pi)^{-N / 2} \gamma_{\boldsymbol{n}_{l}}^{N / 2} \\
& \quad \exp \left[-\gamma_{\boldsymbol{n}_{l}}\left\|\boldsymbol{g}_{l}-\boldsymbol{H} \boldsymbol{M}_{l} \boldsymbol{f}\right\|^{2} / 2\right] \\
= & (2 \pi)^{-N / 2} \gamma_{\boldsymbol{n}_{l}}{ }^{N / 2} \\
& \quad \exp \left[-\gamma_{\boldsymbol{n}_{l}}\left\|\stackrel{\circ}{\boldsymbol{g}}_{l}-\boldsymbol{\Lambda}_{\boldsymbol{H}} \boldsymbol{F} \boldsymbol{M}_{l} \boldsymbol{f}\right\|^{2} / 2\right]
\end{aligned}
$$

thanks to the Parseval relationship, with $\stackrel{\circ}{=}=\boldsymbol{F g}$. The law for all the images is

$$
\begin{aligned}
& p\left(\boldsymbol{g} \mid \boldsymbol{f}, \boldsymbol{\gamma}_{\boldsymbol{n}}\right)=(2 \pi)^{-N L / 2}|\boldsymbol{\Sigma}|^{-1 / 2} \\
& \quad \exp \left[-\frac{1}{2}(\boldsymbol{g}-\overline{\boldsymbol{H}} \boldsymbol{M} \boldsymbol{R} \boldsymbol{f})^{t} \boldsymbol{\Sigma}_{\boldsymbol{n}}^{-1}(\boldsymbol{g}-\overline{\boldsymbol{H}} \boldsymbol{M} \boldsymbol{R} \boldsymbol{f})\right]
\end{aligned}
$$

where $\boldsymbol{\Sigma}_{\boldsymbol{n}}^{-1}$ is block diagonal and each block is $\gamma_{\boldsymbol{n}_{k}} \boldsymbol{I}$. If the noise power is considered to have the same value for each image, then $\boldsymbol{\Sigma}_{\boldsymbol{n}}^{-1}=\gamma_{\boldsymbol{n}} \boldsymbol{I}$ and the data law is

$$
\begin{aligned}
p\left(\boldsymbol{g} \mid \boldsymbol{f}, \gamma_{\boldsymbol{n}}\right)=(2 \pi)^{-N L / 2} \gamma_{\boldsymbol{n}}{ }^{N L / 2} & \\
& \exp \left[-\gamma_{\boldsymbol{n}}\|\boldsymbol{g}-\overline{\boldsymbol{H}} \boldsymbol{M} \boldsymbol{R} \boldsymbol{f}\|^{2} / 2\right] .
\end{aligned}
$$

\section{Prior law of the HR image}

The definition of the prior law for the HR image gives an opportunity to introduce additional information, e.g. a positivity constraint (not used in this paper). Since our forward model includes a convolution operator, some information on the smoothness features of the image must be introduced to cancel the amplification of noise, and to that goal, we use Gaussian fields.

The probability law for the HR image with a given covariance matrix $\Sigma_{\gamma}$ parametrized by a vector $\gamma$ reads

$$
p(\boldsymbol{f} \mid \boldsymbol{\gamma})=(2 \pi)^{-N / 2}\left|\boldsymbol{\Sigma}_{\boldsymbol{\gamma}}\right|^{-1 / 2} \exp \left[-\frac{1}{2} \boldsymbol{f}^{t} \boldsymbol{\Sigma}_{\boldsymbol{\gamma}} \boldsymbol{f}\right] .
$$

For computational efficiency, the precision matrix is designed (or approximated) in a toroidal manner, and is diagonal in the Fourier domain $\Lambda_{f}=\boldsymbol{F} \boldsymbol{\Sigma}_{\gamma} \boldsymbol{F}^{\dagger}$. Thus, the law for $\boldsymbol{f}$ writes

$$
\begin{aligned}
p\left(\boldsymbol{f} \mid \gamma_{\boldsymbol{f}}\right)=(2 \pi)^{-N / 2} \operatorname{det}[\boldsymbol{F}] \operatorname{det}\left[\boldsymbol{\Lambda}_{\boldsymbol{f}}\right]^{1 / 2} \operatorname{det}\left[\boldsymbol{F}^{\dagger}\right] \\
\exp \left[-\frac{1}{2} \boldsymbol{f}^{t} \boldsymbol{F}^{\dagger} \boldsymbol{\Lambda}_{\boldsymbol{f}} \boldsymbol{F} \boldsymbol{f}\right] \\
=(2 \pi)^{-N / 2} \operatorname{det}\left[\boldsymbol{\Lambda}_{\boldsymbol{f}}\right]^{1 / 2} \exp \left[-\frac{1}{2} \stackrel{\circ}{\boldsymbol{f}}^{\dagger} \boldsymbol{\Lambda}_{\boldsymbol{f}} \boldsymbol{f}\right] .
\end{aligned}
$$

This approximation is sometimes referred to as a Whittle approximation [21] (see also [22] or [23]) for the Gaussian law.

In this paper, we focus mostly on smooth images, that can be modeled by positive correlations between pixels. This can be introduced by high-frequency penalties using any circulant differential operator (like Laplacian). The differential operator is denoted by $\boldsymbol{D}$ and its diagonalized form by $\boldsymbol{\Lambda}_{\boldsymbol{D}}=\boldsymbol{F} \boldsymbol{D} \boldsymbol{F}^{\dagger}$. Then, the inverse variance matrix writes $\boldsymbol{\Sigma}_{\gamma_{f}}^{-1}=\gamma_{\boldsymbol{f}} \boldsymbol{D}^{t} \boldsymbol{D}$ and its Fourier counterpart writes $\boldsymbol{\Lambda}_{\boldsymbol{f}}=\gamma_{\boldsymbol{f}} \boldsymbol{\Lambda}_{\boldsymbol{D}}^{\dagger} \boldsymbol{\Lambda}_{\boldsymbol{D}}$ where $\gamma_{\boldsymbol{f}}$ is a positive scale factor tuning the degree of smoothness. The Wiener filter uses the identity matrix $\boldsymbol{I}$ instead of $\boldsymbol{D}$.

In addition to the efficiency of the numerical computation due to Gaussian hypothesis, this model also offers as an advantage the knowledge of the normalization coefficient and its dependency on the hyper-parameter $\gamma_{\boldsymbol{f}}$. With this form of correlation, the law can be written as:

$$
p\left(\boldsymbol{f} \mid \gamma_{\boldsymbol{f}}\right) \propto \gamma_{\boldsymbol{f}}^{(N-1) / 2} \exp \left[-\frac{\gamma_{\boldsymbol{f}}}{2}\left\|\boldsymbol{\Lambda}_{\boldsymbol{D}} \stackrel{\mathrm{f}}{\mathrm{f}}\right\|^{2}\right] .
$$

The parameters $\gamma_{n}$ and $\gamma_{\boldsymbol{f}}$ are usually tuned empirically. The next section presents a way to determine them automatically.

\section{Prior law of hyper parameters}

The parameters $\gamma_{n}$ and $\gamma_{\boldsymbol{f}}$ are considered unknown. With respect to the Bayesian framework

$$
p\left(\boldsymbol{f}, \boldsymbol{\gamma}_{\boldsymbol{n}}, \gamma_{\boldsymbol{f}} \mid \boldsymbol{g}\right) \propto p\left(\boldsymbol{f} \mid \boldsymbol{g}, \boldsymbol{\gamma}_{\boldsymbol{n}}\right) p\left(\boldsymbol{f} \mid \gamma_{\boldsymbol{f}}\right) p\left(\boldsymbol{\gamma}_{\boldsymbol{n}}\right) p\left(\gamma_{\boldsymbol{f}}\right)
$$

prior laws $p\left(\boldsymbol{\gamma}_{\boldsymbol{n}}\right)$ and $p\left(\gamma_{\boldsymbol{f}}\right)$ for the parameters must be defined. A classical choice for hyper parameter laws relies on conjugate prior [24]: the conditional posterior for the hyperparameters is in the same family as its prior. All the parameters are precision parameters of a Gaussian law and a conjugate 
law for these parameters is the Gamma law. Given parameters $\left(\alpha_{i}, \beta_{i}\right)$ the pdf reads

$$
\begin{aligned}
p\left(\gamma_{i}\right) & =\mathcal{G}\left(\alpha_{i}, \beta_{i}\right) \\
& =\frac{1}{\beta_{i}^{\alpha_{i}} \Gamma\left(\alpha_{i}\right)} \gamma_{i}^{\alpha_{i}-1} \exp \left(-\gamma_{i} / \beta_{i}\right), \forall \gamma_{i} \in[0,+\infty[
\end{aligned}
$$

Very little prior information is available for these parameters. With parameters set to $(0,+\infty)$ values, one obtains the Jeffreys' non-informative prior. Jeffreys' law is a classical law for the precisions and is considered as non-informative [25].

\section{E. Posterior mean estimator}

The joint posterior law is defined as

$$
\begin{aligned}
& p\left(\boldsymbol{f}, \gamma_{\boldsymbol{n}}, \gamma_{\boldsymbol{f}} \mid \boldsymbol{g}\right) \propto \gamma_{\boldsymbol{f}}{ }^{(N-1) / 2-1} \prod_{l=0}^{L-1}\left[\gamma_{\boldsymbol{n}_{l}}{ }^{N / 2-1}\right] \\
& \exp \left[-\sum_{l=0}^{L-1} \frac{\gamma_{\boldsymbol{n}_{l}}}{2}\left\|\boldsymbol{g}_{l}-\boldsymbol{H} \boldsymbol{M}_{l} \boldsymbol{f}\right\|^{2}\right] \exp \left[-\frac{\gamma_{\boldsymbol{f}}}{2}\|\boldsymbol{D} \boldsymbol{f}\|^{2}\right] .
\end{aligned}
$$

This law is multidimensional (the number of dimensions is the number of pixels in the HR image along with the number of unknown hyper-parameters) and quite complex. Clearly, it is not possible to manipulate or keep all the information embedded in the posterior law. The classical solution relies on the choice of a particular point like the point of maximum probability called maximum a posteriori (MAP)

$$
\left[\widehat{\boldsymbol{f}}, \widehat{\gamma_{\boldsymbol{n}}}, \widehat{\gamma_{\boldsymbol{f}}}\right]=\underset{\boldsymbol{f}, \boldsymbol{\gamma}_{\boldsymbol{n}}, \gamma_{\boldsymbol{f}}}{\arg \max } p\left(\boldsymbol{f}, \boldsymbol{\gamma}_{\boldsymbol{n}}, \gamma_{\boldsymbol{f}} \mid \boldsymbol{g}\right)
$$

that leads to an optimization problem, or the mean of the law

$$
\left[\widehat{\boldsymbol{f}}, \widehat{\gamma_{\boldsymbol{n}}}, \widehat{\gamma_{\boldsymbol{f}}}\right]=\int\left[\boldsymbol{f}, \boldsymbol{\gamma}_{\boldsymbol{n}}, \gamma_{\boldsymbol{f}}\right] p\left(\boldsymbol{f}, \boldsymbol{\gamma}_{\boldsymbol{n}}, \gamma_{\boldsymbol{f}} \mid \boldsymbol{g}\right) \mathrm{d} \boldsymbol{f} \mathrm{d} \boldsymbol{\gamma}_{\boldsymbol{n}} \mathrm{d} \gamma_{\boldsymbol{f}}
$$

that leads to an integration problem. Both estimators are possible, but the posterior mean has some advantages. First, the mean is known to minimize the mean square error (MSE) [24]. Second, use of classical algorithms to compute the mean allows access to all the other moments of the law, including the uncertainty of the estimate. For these reasons, the mean is computed with the help of a Gibbs algorithm.

\section{F. Gibbs sampler}

To compute the posterior mean, a Monte Carlo Markov chain is used to provide stochastic samples. The provided samples allow computing moments of the law, like the mean and the standard deviation, assimilated to uncertainty on the mean. Afterwards, samples are also used to approximate marginal laws as histograms.

The Gibbs algorithm [24], [26], [27], first introduced in image processing by Geman \& Geman [28], is an MCMC algorithm to sample any law. It relies on iterative sampling of the conditional posterior law for a set of parameters given the other obtained through previous iterations. The structure of the algorithm is a repetition of following steps:
1) Draw $\boldsymbol{f} \sim p\left(\boldsymbol{f} \mid \boldsymbol{\gamma}_{\boldsymbol{n}}{ }^{(k)}, \gamma_{\boldsymbol{f}}{ }^{(k)}, \boldsymbol{g}\right)$, which is a multidimensional Gaussian law. The size and complexity of the covariance law prevents direct sampling. Classical algorithms like per-pixel sampling [28] or Cholesky factorisation [29], [30] are impossible in practice because of either the required time or of memory. The appendix A proposes an adapted algorithm of [29], [30] approaches based on the resolution of an optimization problem.

2) Draw $\gamma_{\boldsymbol{n}_{l}} \sim p\left(\gamma_{\boldsymbol{n}_{l}} \mid \boldsymbol{f}^{(k+1)}, \gamma_{\boldsymbol{f}}^{(k)}, \boldsymbol{g}\right)$, which is a Gamma law

$$
p\left(\gamma_{\boldsymbol{n}_{l}} \mid \boldsymbol{f}^{(k+1)}, \boldsymbol{g}\right)=\mathcal{G}\left(N L / 2,2 /\left\|\boldsymbol{g}_{l}-\boldsymbol{H} \boldsymbol{M}_{l} \boldsymbol{f}^{(k+1)}\right\|^{2}\right),
$$

3) Draw $\gamma_{\boldsymbol{f}} \sim p\left(\gamma_{\boldsymbol{f}} \mid \boldsymbol{f}^{(k+1)}, \gamma_{\boldsymbol{n}}{ }^{(k+1)}, \boldsymbol{g}\right)$, also a Gamma law

$$
p\left(\gamma_{\boldsymbol{f}} \mid \boldsymbol{f}^{(k+1)}\right)=\mathcal{G}\left((N-1) / 2,2 /\left\|\boldsymbol{D} \boldsymbol{f}^{(k+1)}\right\|^{2}\right) .
$$

After a burn-in or convergence time, the chain becomes stationary and the complete set of samples $\left\{\boldsymbol{f}^{(k)}, \gamma_{\boldsymbol{f}}{ }^{(k)}, \boldsymbol{\gamma}_{\boldsymbol{n}}{ }^{(k)}\right\}_{k \in \mathcal{K}}$ follows the joint posterior law, Eqn. (27). Then, the posterior mean is approximated by an average of the samples

$$
\widehat{\boldsymbol{f}}=\mathbb{E}[\boldsymbol{f}] \approx \frac{1}{K} \sum_{k=0}^{K-1} \boldsymbol{f}^{(k)}
$$

using the large numbers law.

In addition to the mean, the samples can be used to compute other moments of the posterior law. For example, the posterior variance:

$$
\mathbb{V}[\boldsymbol{f}]=\mathbb{E}\left[(\boldsymbol{f}-\mathbb{E}[\boldsymbol{f}])(\boldsymbol{f}-\mathbb{E}[\boldsymbol{f}])^{t}\right]
$$

is used to provide uncertainty about the estimated value. In the case of the image, the covariance matrix is very large, but the diagonal value (variance of each pixel) is obtained with

$$
\widehat{\mathbb{V}}[\boldsymbol{f}] \approx \frac{1}{K} \sum_{k=0}^{K-1}\left[\boldsymbol{f}^{(k)} \odot \boldsymbol{f}^{(k)}\right]-\widehat{\boldsymbol{f}}^{2}
$$

where $\odot$ is the term-wise multiplication.

\section{NUMBER OF LR IMAGES}

In the standard SIM approach [1], [13], each sub-pattern in Fig. 1 is considered to be a different unknown. The particular illumination structure with $2 \pi / 3$ phase shifting allows the resolution of the system simply by linear combination between nine images. The idea to build an equation system that takes into account the redundancy in the data and achieves a reduction of the number of required images is presented in [31]. In that paper, the possibility to reduce the number of images down to four images in linear SIM is already stated and thought of, however without specific implementation details and examples.

Here, thanks to an inverse problem approach, we prove an effective realisation of the use of redundancy and show that in some conditions and depending on the optical setup, the number of images can indeed be decreased to as low as four. 
The rationale is based on several points. First, in the standard approach, the $D_{0}$ sub-pattern is reconstructed three times, once for each orientation, although it is the same image in all three cases ${ }^{3}$ In our proposed approach,it is not necessary to acquire additional images, because the presence of $D_{0}$ is naturally taken into account by the joint inversion of all the data. This effectively allows to reduce the number of image to 7 .

Second, the original image is real, and consequently the pattern $F_{+}$and $F_{-}$, in Eq. (5), are identical up to a conjugacy. Moreover, the satellite and central patterns are equal on some interval $F_{+}\left(f_{m}-f\right)=F^{*}(f)$. Consequently, as illustrated in Fig. 3. as long as $f_{m} \leq f_{c}$, there are only two unknowns inside the support of the OTF : $F(f), \forall f \in\left[0, f_{c}\right]$ and $F(f), \forall f \in$ $\left[f_{c}, f_{c}+f_{m}\right]$, if and only if $f_{m} \leq f_{c}$. In Fig. 3 this illustrated by $U_{0}$ and $U_{1}$ being identical.

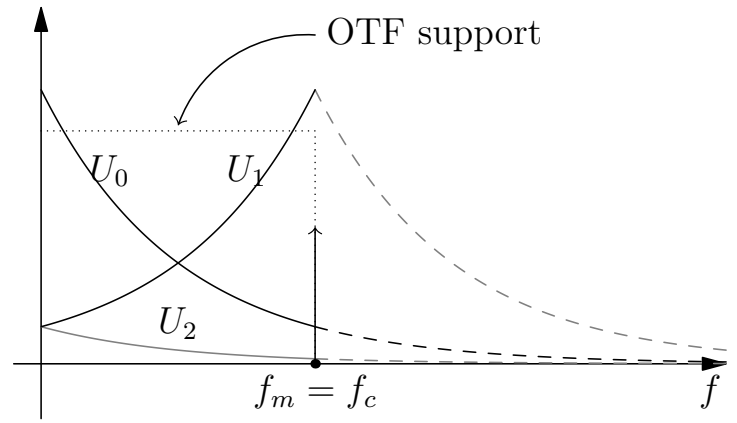

Fig. 3. Modulation of a 1D signal. Because of the conjugacy, only halffrequency planes are shown.

The extension to 2D is straightforward. Eqn. (6) can be rewritten as

$G\left(f_{x}, f_{y}\right)=D_{0}\left(f_{x}, f_{y}\right)+D_{+}\left(f_{x}, f_{y}\right)+D_{+}^{*}\left(f_{x}-2 k_{x}, f_{y}-2 k_{y}\right)$

and for each orientation, there are only two orders, $D_{0}$ and $D_{+}$, instead of three. For one orientation we have exactly two unknowns if $\sqrt{k_{x}^{2}+k_{y}^{2}} \leq f_{c}$, as illustrated in Fig. 4a. except for the surfaces in gray where three unknowns are present. When tiling the plane with images acquired with different orientations, some frequencies can be resolved, as illustrated in Fig. 4b. Frequencies where further ambiguities remain can be estimated thereafter with the information coming both from the likelihood (which is not sufficient by itself ) and the prior law.

To solve the image reconstruction problem with a lower data redundancy, the approach is to have an equation system that considers all the data jointly to estimate all the unknowns, without ambiguity, as discussed in [31]. Our proposed inversion approach, which uses a direct model and all the data jointly to reconstruct the image in one global step, is an implementation of the latter. If we consider, for the sake of simplicity, the same noise level for all image, then the step 1)

\footnotetext{
${ }^{3}$ This is true when a sufficiently high density of photons with random polarisation are acquired, leading to an indistinguishable polarisation. This was the case for our data: we had a minimum of 450 photons per pixel, and the conditions were met. It should be noted that birefringent samples would however be a limitation of the setup.
}

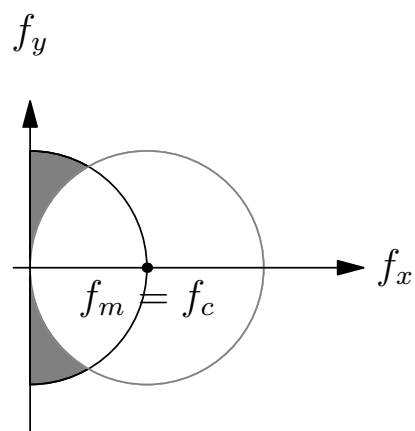

(a)

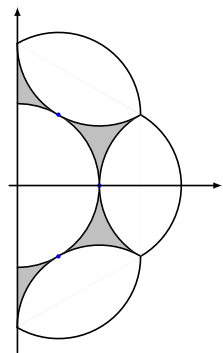

(b)

Fig. 4. Modulation of a $2 \mathrm{D}$ signal. (a) Only two unknowns $\left(U_{0}, U_{1}\right)$ are present in the surface $\sqrt{f_{x}^{2}+f_{y}^{2}} \leq f_{c}$ except in the gray parts where three unknowns are present; (b) The gray areas represent the frequencies where the likelihood alone is not sufficient to resolve the ambiguity in the final reconstruction.

in the algorithm (Sec. III-F) corresponds to the simulation of the conditional posterior law:

$$
p\left(\boldsymbol{f} \mid \boldsymbol{g}, \boldsymbol{\gamma}_{\boldsymbol{n}}, \gamma_{\boldsymbol{f}}\right) \propto \exp \left[-\frac{\gamma_{\boldsymbol{n}}}{2}\|\boldsymbol{g}-\boldsymbol{H} \boldsymbol{M} \boldsymbol{f}\|^{2}-\frac{\gamma_{\boldsymbol{f}}}{2}\|\boldsymbol{D} \boldsymbol{f}\|^{2}\right]
$$

The Gaussian law can be handled through a quadratic criterion (see Appendix. A)

$$
J(\boldsymbol{f}) \propto \gamma_{\boldsymbol{n}}\|\boldsymbol{g}-\boldsymbol{H} \boldsymbol{M} \boldsymbol{f}\|^{2}+\gamma_{\boldsymbol{f}}\|\boldsymbol{D} \boldsymbol{f}\|^{2} .
$$

where the optimisation leads to the resolution of the equation system

$$
\left(\boldsymbol{M} \boldsymbol{H} \boldsymbol{H}^{t} \boldsymbol{M}^{t}+\boldsymbol{D}^{t} \boldsymbol{D}\right) \boldsymbol{f}=\boldsymbol{M}^{t} \boldsymbol{H}^{t} \boldsymbol{g}
$$

effectively solved with a conjugated gradient descent algorithm (see [32] and Appendix. A. In the equation system Eq. (34) all the pixels of the image $f$ are considered jointly with all the data collected in $\boldsymbol{g}$.

\section{EXPERIMENTS}

In this section, the proposed method for super-resolution imaging with structured illumination is studied. The study focuses mainly on image quality and on the contributions of the proposed approach.

\section{A. Simulations}

The experiments are conducted on two simulated cases, "Barbara" and the "test pattern", well suited to analyze the improvement in resolution and on real data obtained with "Structured Illumination Microscopy" [1], [12], [13]. The results are also compared with a classical deconvolution approach [13], [22].

The data has been simulated by our forward model described in Sec. III-A Without loss of generality, the PSF is the classical Airy disc often encountered in optical problems [11]. Its expression in Fourier space is

$$
H(f)=\frac{1}{\pi}\left[2 \cos ^{-1}\left(\frac{f}{f_{c}}\right)-\sin \left(2 \cos ^{-1}\left(\frac{f}{f_{c}}\right)\right)\right],
$$

$\forall|f| \in\left[0, f_{c}\right], 0$ otherwise. With this PSF, the cutoff frequency is at $f_{c}$, beyond which no information is available. 
The modulation patterns are described in Sec. [-B Eq. (1). The number of LR images is four with three different orientations to construct a "second ring" of frequency. The orientations are $-30,30$ and 90 degrees. In all cases, the reconstruction of the HR image is done with only four LR images: one without structured illumination and three with illumination in the three different orientations.

The regularity of the reconstructed image is measured through the Laplacian $\boldsymbol{D}=[0,-1,0 ;-1,4,-1 ; 0,-1,0]$ and $\boldsymbol{\Lambda}_{\boldsymbol{D}}$ is obtained with an FFT-2D. Regarding the parameters of the Gamma laws, we set $\alpha$ and $\beta$ to $(0,+\infty)$ in order to choose the non-informative Jeffreys' law [25].

The algorithm used is described in Sec. III-F A burn-in period of 20 samples is set before the computation of the empirical mean by Eqn. (30). The algorithm 1 is stopped following the criterion

$$
\left\|\widehat{\boldsymbol{f}}^{(K+1)}-\widehat{\boldsymbol{f}}^{(K)}\right\|^{2} /\left\|\widehat{\boldsymbol{f}}^{(K)}\right\|^{2} \leq \epsilon
$$

when the difference between two successive means is less than $\epsilon=10^{-3}$, where $K$ is total number of samples.

The results are compared to the classical approach [13] that requires nine LR images. To observe the improvement in image quality provided by structured illumination, the results are also compared to a multichannel unsupervised deconvolution approach like [22] with four channels (4 LR images). Since the noise model is white Gaussian, this corresponds to a deconvolution with 1 LR image and $4 \gamma_{n}$ higher precision level.

The objective metrics used to quantify the quality of the results are (i) the improved signal-to-noise ratio (ISNR) of estimate $\widehat{\boldsymbol{f}}_{1}$ over $\widehat{\boldsymbol{f}}_{2}$ defined as

$$
\operatorname{ISNR}\left(\widehat{\boldsymbol{f}}_{1}, \widehat{\boldsymbol{f}}_{2}\right)=10 \log _{10}\left(\left\|\boldsymbol{f}-\widehat{\boldsymbol{f}}_{2}\right\|^{2} /\left\|\boldsymbol{f}-\widehat{\boldsymbol{f}}_{1}\right\|^{2}\right)
$$

and (ii) the SSIM (Structural SIMilarity) [33] designed to be adapted to human visual perception

$$
\operatorname{SSIM}\left(\widehat{\boldsymbol{f}}_{1}, \widehat{\boldsymbol{f}}_{2}\right)=\frac{\left(2 \mu_{1} \mu_{2}+c_{1}\right)\left(2 \operatorname{cov}_{12}+c_{2}\right)}{\left(\mu_{1}^{2}+\mu_{2}^{2}+c_{1}\right)\left(\sigma_{1}^{2}+\sigma_{y}^{2}+c_{2}\right)}
$$

where $\mu$ and $\sigma$ are the mean and standard deviation of the vectors, $\operatorname{cov}_{12}$ the covariance between $\widehat{\boldsymbol{f}}_{1}$ and $\widehat{\boldsymbol{f}}_{2}$ and $c_{1}$ and $c_{2}$ are stabilisation terms. The SSIM index is a measure between an image and a reference (ground truth here) and takes its value between -1 and 1 ( 1 meaning full identity with the reference).

The algorithm has been applied on two sets of simulated data:

- The "Barbara" image, Fig. 5a, which is a $519 \times 519$ pixel image. The cutoff frequency is set to 0.3 in reduced frequency, and therefore almost all the frequency plane of the original sampled image can be observed. In this simulated image, frequencies above 0.5 are not present and therefore no expansion of the frequencies plane is required.

- The "test pattern" image, Fig. 5b which is a $263 \times 263$ pixel image. The cutoff frequency is set to 0.12 in reduced frequency.

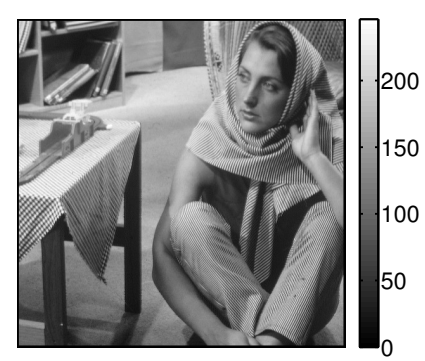

(a) Barbara

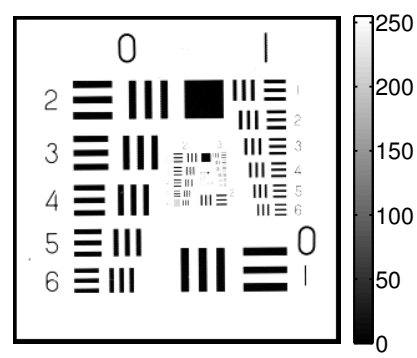

(b) Test pattern
Fig. 5. True images used for the study (in arbitrary units).

The tests have been conducted with the following conditions:

- $\mathrm{SNR} \approx 80 \mathrm{~dB}$. This is a favorable case, with standard deviation $\sigma \approx 1$ for an intensity range in $[0,255]$, and approximately 6500 photons per pixel (for real data acquired with an ANDOR iXon 885 camera and a shotnoise model), and

- $\mathrm{SNR} \approx 20 \mathrm{~dB}$. This is an unfavorable case, with $\sigma \approx 6$ and a number of photons of approximately 450 per pixel, and where the same noise level is applied to all LR images. The real data present an SNR in the range 20 to $40 \mathrm{~dB}$.

1) Estimation results: Results on "Barbara" are illustrated in Fig. 6. For comparison, the Fig. 6a is the data obtained with simulated brightfield illumination (without structured illumination) and 6e is a slice. They are the results of a simulated convolution with an optical system. Compared to the true image 5a, small details are no longer visible, especially, the stripes on clothes. Results from deconvolution are illustrated in 6b and 6f. Some details are restored around pixel 150, but the stripes are still not visible. Results from the structured illumination with the proposed approach are illustrated in Figs. 6c and 6g. With this approach, many more small details are visible. The stripes on clothes are visible and high frequencies around pixel 25 or 225 are clearly restored in comparison to the deconvolution. Our results are virtually identical to the classical approach [13], illustrated Figs. 6d] and $6 \mathrm{~h}$. where 9 images are necessary.

This gain in high frequencies is clearly visible in the "spectrum" illustrated in Fig. 7. Since the optical transfer function is zero outside $f_{c}$, the deconvolution process is unable to restore frequencies outside this limit, as shown by Fig. $7 \mathrm{c}$ The structured illumination, through modulation, is able to recover high frequencies above $f_{c}$. This is well illustrated by Fig. 7d. The tiling pattern in the three orientations is visible. A close resemblance with the true spectrum in Fig. $7 \mathrm{a}$ can be noticed, as well as the presence of the high-frequencies necessary for reconstructing the stripes on clothes.

The fact that high frequencies are measured beyond the cutoff frequency $f_{c}$ of the optical transfer function is illustrated in the empirical estimation of the circular mean of the spectrum in Fig. $8 \mathrm{a}$ The gain in low frequency with deconvolution is visible only up to $f_{c}$, while It can be seen that structured illumination allows restoring frequencies well beyond that limit. In this experiment, the level of noise is low enough 


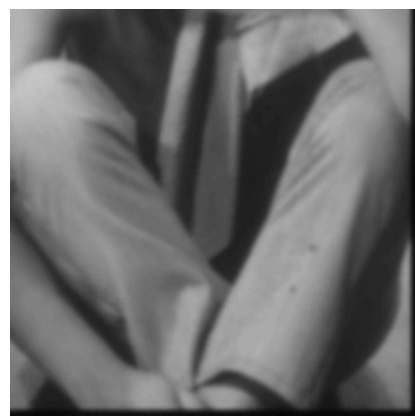

(a) Data

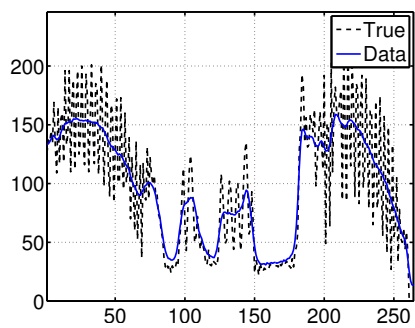

(e) Line of data

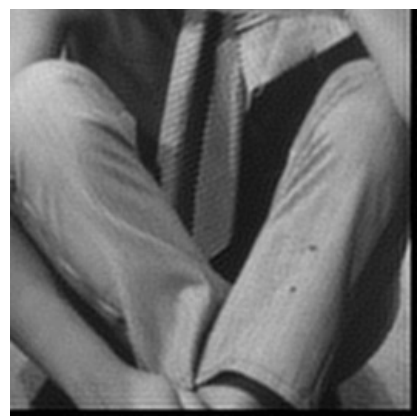

(b) Deconvolution

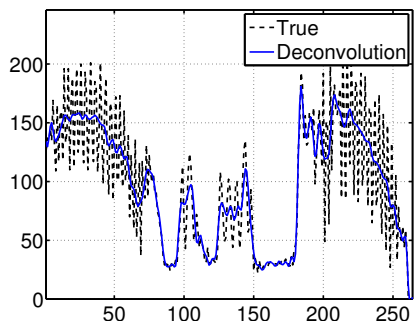

(f) Line of deconvolution

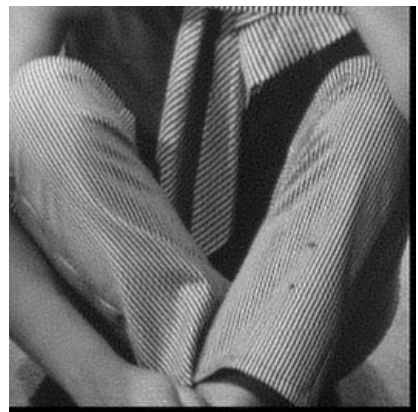

(c) Proposed

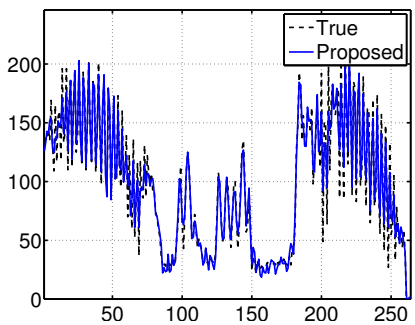

(g) Line of proposed

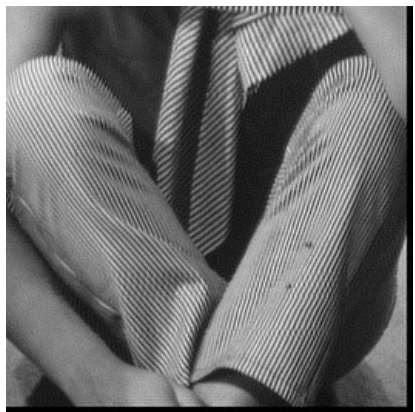

(d) Classical approach [13]

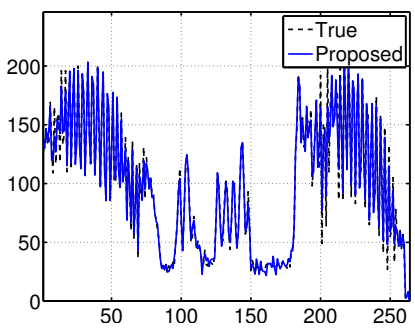

(h) Line of 13

Fig. 6. Comparison of reconstruction quality on "Barbara" with SNR $\approx 80 \mathrm{~dB}$. The x-axis of slice images gives the pixel number. The improvement achieved by structured illumination over deconvolution is clearly noticeable. The result of our SI approach with 4 images is very close to the classical SI approach with 9 images.

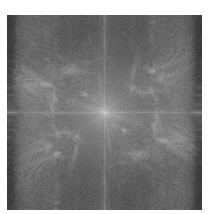

(a) True

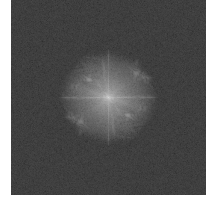

(b) Data

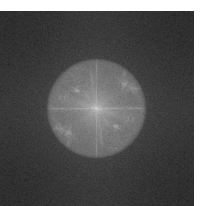

(c) 22

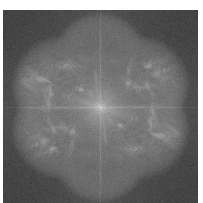

(d) Proposed

Fig. 7. Spectrum of "Barbara". Figures are the logarithm of the absolute value of FFT-2D.

to allow a high quality restoration up to frequencies close to the highest one.

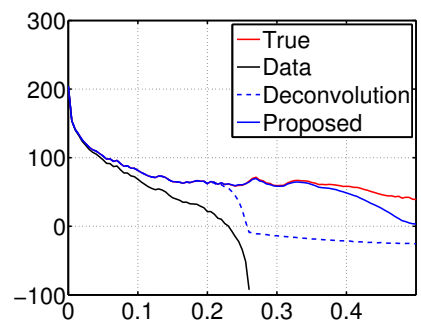

(a) Barbara

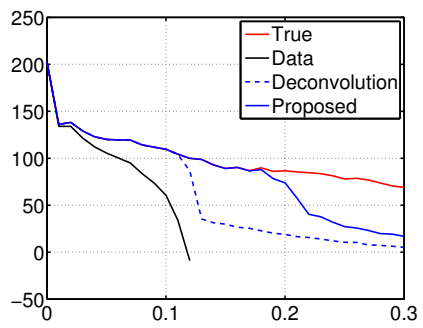

(b) Test pattern

Fig. 8. Empirical estimation of circular power spectral density. The x-axis is the reduced frequency. The $y$-axis is the square value of the absolute value of Fourier transform in $\mathrm{dB}$.

Results with the "test pattern" in Fig. 9 are similar, notwithstanding lower modulation and cutoff frequencies, meaning that less high frequencies are measured. In comparison to the deconvolution result in Fig. 9b, the structured illumination in Fig. $9 \mathrm{c}$ increases the spatial resolution and visibility of small details, which is also obvious from the spectrum (Fig. 10)

with the tiling. On the circular mean (Fig. 8b), it is clear that frequencies above $f_{c} \approx 0.11$ and up to $f_{c}+f_{m} / 2 \approx 0.18$ are restored.

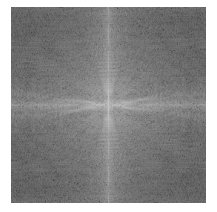

(a) True

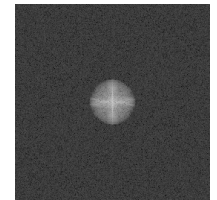

(b) Data

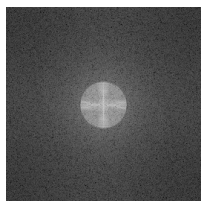

(c) $\mid 22$

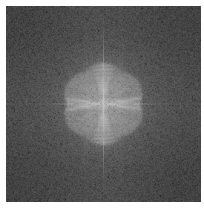

(d) Proposed
Fig. 10. Spectrum of "test pattern". Figures are the logarithm of the absolute value of FFT-2D.

Numerical performances are reported in Tab. I For a noise level corresponding to an SNR $\approx 80 \mathrm{~dB}$, for "Barbara", the gain in ISNR for structured illumination is more than threefold, 7.12 in comparison to the deconvolution with 1.68 (for the same amount of data). In the case of "test pattern" too, the gain is significant, 6.1 for structured illumination and 3.5 for deconvolution. In both cases, there is an improvement in the SSIM index from 0.75 for the deconvolution to 0.85 for the structured illumination. The quantitative improvement of the classical approach in all cases is easily explained by the use of more than double the number of images than with our approach, reducing the influence of noise.

When the noise level is quite high $(\mathrm{SNR} \approx 20 \mathrm{~dB}$ ), the results may seem quite disappointing. Indeed, although an improvement over raw data is visible, the performances of SI are not much higher than those of the deconvolution. This can be explained by the fact that inside the support of the optical transfer function, the white noise corrupts the aliased high frequencies coming from the modulation. In other words, even 


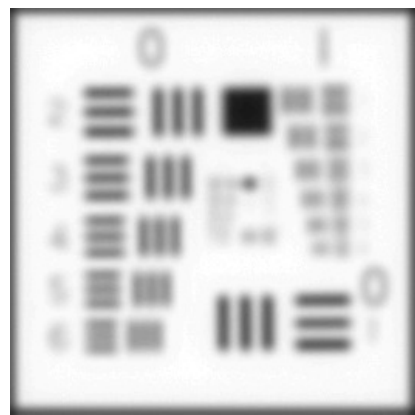

(a) Data

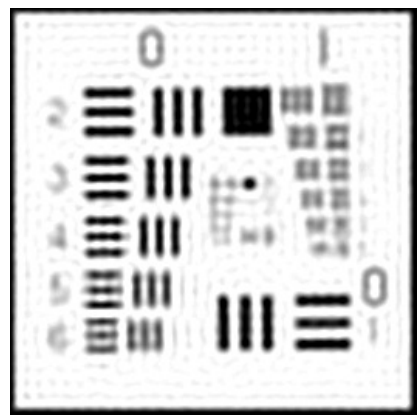

(b) Deconvolution

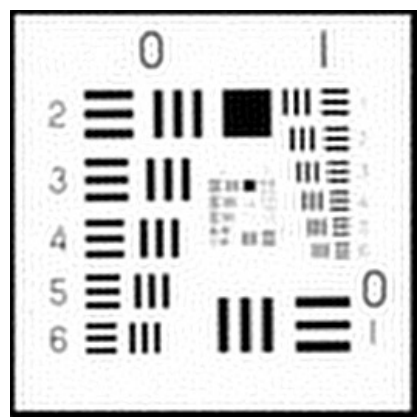

(c) Proposed

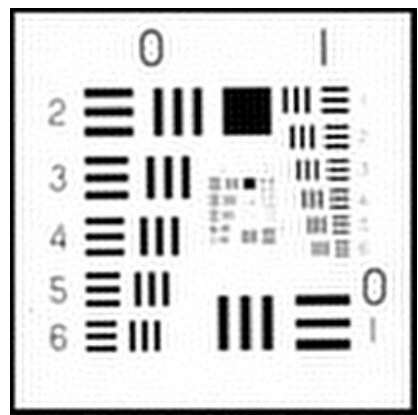

(d) Classical approach [13]

Fig. 9. Comparison of reconstruction quality on "test pattern". The improvement achieved through Structured Illumination with 9 or 4 images is clear.

if high frequencies are aliased inside the support of the transfer function, because their level is low, they are indistinguishable from the noise.

\begin{tabular}{l|c|cccc} 
& $\approx \mathrm{SNR}$ & & ISNR & SSIM & $\hat{\sigma}$ \\
\hline Barbara & $80 \mathrm{~dB}$ & Data & - & 0.67 & - \\
& & {$[22]$} & 1.68 & 0.75 & - \\
& & Proposed & 1.78 & 0.21 & 9.3 \\
& & {$[13]$} & 1.12 & 0.10 & 7.4 \\
\hline \multirow{5}{*}{ test pattern } & $20 \mathrm{~dB}$ & Data & - & 0.44 & - \\
& & {$[22]$} & 1.43 & 0.64 & - \\
& & Proposed & 0.38 & 0.16 & 8.4 \\
& & {$[13]$} & 0.22 & 0.08 & 7.7 \\
\hline & & Data & - & 0.55 & - \\
& & Proposed & 1.55 & 0.16 & 14.6 \\
& & {$[13]$} & 0.72 & 0.08 & 12.1 \\
\hline & $20 \mathrm{~dB}$ & Data & - & 0.31 & - \\
& & {$[22]$} & 2.79 & 0.44 & - \\
& & Proposed & 0.93 & 0.11 & 28.9 \\
& & {$[13]$} & 0.54 & 0.06 & 25.6
\end{tabular}

NUMERICAL EVALUATIONS OF PERFORMANCE. ISNR AND SSIM ARE THE GAIN PER LR IMAGES USED. DATA CORRESPOND TO LR IMAGES OBTAINED WITHOUT STRUCTURED ILLUMINATION (SI). [22] IS THE DECONVOLUTION, "PROPOSED" IS OUR RECONSTRUCTION WITH ONLY 4 IMAGES AND [13] THE CLASSICAL SI APPROACH WITH 9 IMAGES. QUANTITATIVE PERFORMANCE IS SLIGHTLY BETTER WITH |13| BECAUSE OF THE USE OF MORE THAN DOUBLE THE NUMBER OF IMAGES FOR THE SAME QUALITATIVE RESULT.

As described in Sec. II-E, the proposed approach allows to compute the uncertainty about the estimated image through the standard deviation of the posterior law. Fig. 11 illustrates the estimated uncertainty with the "test pattern" with $\mathrm{SNR} \approx 80$ $\mathrm{dB}$, while Tab. I reports the mean value. In this experiment, the uncertainty $\hat{\sigma}$ has an average value of $\approx 15$ for a signal value between 0 and 255. A very striking fact is the structure of the uncertainty (Fig. 11a) in relation to the illumination pattern (Fig. 11b). Clearly, there is a strong correlation between the illumination and the uncertainty patterns: a higher illumination of the sample, i.e. more photons being shone on and emitted from the sample, is associated with a lesser uncertainty on the reconstruction, and conversely when there are less photons, the uncertainty is higher. While this is true when working with simulated data with a stationary Gaussian noise model, it may differ in the case of a non stationary noise model like Poisson.
As can be seen from Tab. II, the use of more than double images makes the classical SI approach slightly less sensitive to noise with a reduced uncertainty.

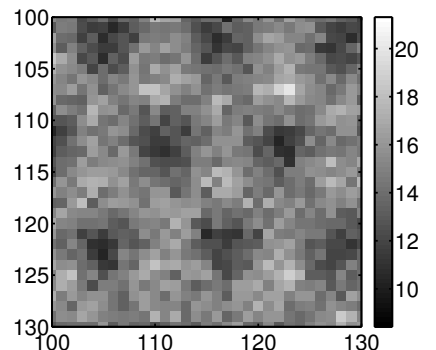

(a) Uncertainty

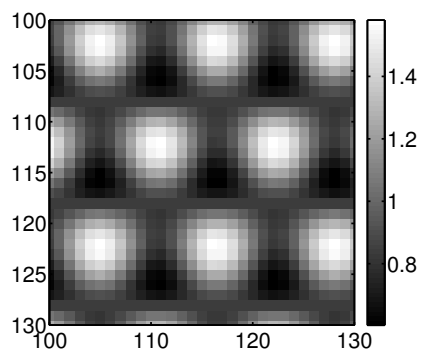

(b) Illumination
Fig. 11. Uncertainty about the estimate for simulated data and a stationary Gaussian noise model. Fig. 11a is the posterior standard deviation (in image unit), see Eqn. (32) of the "test pattern" with SNR $\approx 80 \mathrm{~dB}$. Fig. $11 \mathrm{~b}$ is the mean of all illumination patterns (without unit). Clearly, there is less uncertainty at locations where there is more illumination. Both axis are pixel numbers.

2) Hyper-parameters estimation: Fig. 12 illustrates the Markov chains and histograms of the noise and image power for the "Barbara" experiment with $\mathrm{SNR} \approx 80 \mathrm{~dB}$. The chains look similar for other experiments.

Numerical estimation results are reported in Tab. III. For the parameter $\gamma_{\boldsymbol{n}}$, the estimations are very close to the true values in all the cases, $\gamma_{\boldsymbol{n}}=0.99$ and $\gamma_{\boldsymbol{n}}=0.0029$ for true values equal to 1 and 0.03 respectively. The uncertainties, provided by the standard deviation of the sample, are small, indicating that the data are informative enough about this parameter. The uncertainty in the case of a SNR $\approx 20 \mathrm{~dB}$ is much lower than in the $80 \mathrm{~dB}$ case. This may be interpreted as the increase in noise power introducing more information about $\gamma_{\boldsymbol{n}}$ value.

As to $\gamma_{f}$, since the true value is not known, the error value (MSE)

$$
e\left(\gamma_{\boldsymbol{f}}\right)=\left\|\boldsymbol{f}-\widehat{\boldsymbol{f}}\left(\gamma_{\boldsymbol{f}}\right)\right\|^{2} /\|\boldsymbol{f}\|^{2}
$$

is computed for several values of $\gamma_{\boldsymbol{f}}$, ranging from $10^{-4}$ to 10 in logarithmic scale, to determine the optimal one for image reconstruction. Results are reported in Tab. II. Globally, the estimated $\hat{\gamma_{f}}$ is in the same range as the optimal one. The uncertainty is small as in the $\gamma_{\boldsymbol{n}}$ case. Consequently, the data are informative enough to automatically tune the trade-off between the likelihood and the spatial regularity. 


\begin{tabular}{|c|c|c|c|c|c|c|c|c|c|}
\hline & SNR & & $\gamma_{n}$ & $\widehat{\gamma_{n}}$ & $\sigma$ & $\gamma_{\boldsymbol{f}}^{*}{ }_{\mathrm{MSE}}$ & $\gamma_{\boldsymbol{f}}^{*}{ }_{\text {SSIM }}$ & $\widehat{\gamma_{f}}$ & $\sigma$ \\
\hline \multirow[t]{4}{*}{ Barbara } & \multirow[t]{2}{*}{$\approx 80 \mathrm{~dB}$} & \multirow{2}{*}{$\begin{array}{c}\text { Proposed } \\
|22|\end{array}$} & 1 & 0.99 & $10^{-3}$ & 0.017 & 0.071 & 0.018 & $10^{-4}$ \\
\hline & & & 1 & 0.99 & $10^{-2}$ & - & - & 0.08 & $6 \times 10^{-6}$ \\
\hline & \multirow[t]{2}{*}{$\approx 20 \mathrm{~dB}$} & \multirow{2}{*}{$\begin{array}{c}\text { Proposed } \\
22 \\
\end{array}$} & 0.03 & 0.0029 & $5 \times 10^{-6}$ & 0.071 & 0.29 & 0.135 & $1.5 \times 10^{-2}$ \\
\hline & & & 0.03 & 0.01 & $3 \times 10^{-5}$ & - & - & 0.154 & $10^{-2}$ \\
\hline \multirow[t]{4}{*}{ test pattern } & \multirow[t]{2}{*}{$\approx 80 \mathrm{~dB}$} & \multirow{2}{*}{$\begin{array}{c}\text { Proposed } \\
{[22]}\end{array}$} & 1 & 0.99 & $3 \times 10^{-3}$ & 0.11 & 1.21 & 0.016 & $2 \times 10^{-4}$ \\
\hline & & & 1 & 1.005 & $2 \times 10^{-2}$ & - & - & 0.012 & $2 \times 10^{-3}$ \\
\hline & \multirow[t]{2}{*}{$\approx 20 \mathrm{~dB}$} & \multirow{2}{*}{$\begin{array}{c}\text { Proposed } \\
|22|\end{array}$} & 0.03 & 0.0029 & $9 \times 10^{-6}$ & 0.0087 & 0.09 & 0.013 & $3 \times 10^{-3}$ \\
\hline & & & 0.03 & & $7 \times 10^{-5}$ & - & - & 0.017 & $5 \times 10^{-3}$ \\
\hline
\end{tabular}

TABLE II

ESTIMATION OF HYPER-PARAMETERs. THE VALUES $\gamma_{\boldsymbol{f}}^{*}$ ARE THE OPTIMAL VALUES WiTH RESPECT TO THE MSE (EQ. 39 OR THE SSIM (EQ. 38 .

The Markov chains and histograms of the parameters are illustrated in Fig. 12 for "Barbara" with an SNR $\approx 80$ dB. Figs. $12 \mathrm{a}$ and $12 \mathrm{~d}$ illustrate the full chains from the initialization on. For both parameters, the burn-in time is approximately 10 samples. Figs. $12 \mathrm{~b}$ and $12 \mathrm{e}$ illustrate the chains from sample 20 to the end. There is a good exploration of the state space around a stable value corresponding to the computed mean. The dispersion of the sample corresponds to the uncertainty. Figs. 12c and 12f are histograms of the sample after the burn-in time. The histograms are concentrated around the stable value and their width corresponds to the uncertainty. These are representations of the marginal posterior law for these parameters.

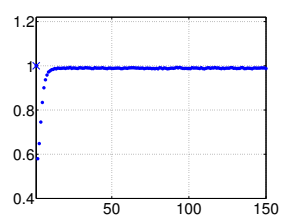

(a)

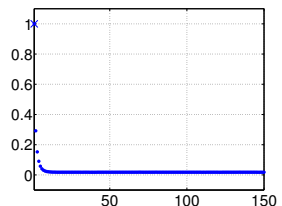

(d)

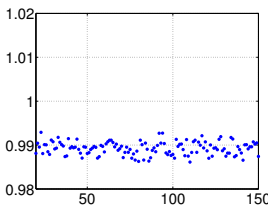

(b)

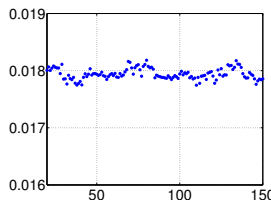

(e)

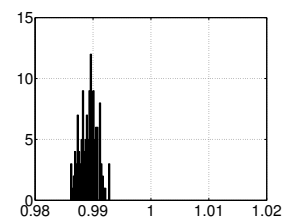

(c)

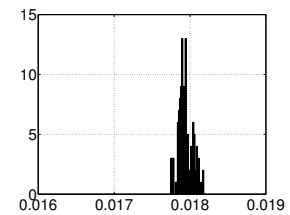

(f)
Fig. 12. Chains of hyper-parameters for "Barbara" with $\mathrm{SNR} \approx 80 \mathrm{~dB}$. The symbol $\times$ denotes the initialization. The $\mathrm{x}$-axis of the chains is iteration and $y$-axis is the precision value (square value of image unit). The $x$-axis of the histograms is the precision value and y-axis is the count of samples.

3) Recovering still higher frequencies with eight images: As noted in section III] our approach allows the use and explotation of any modulation strategy. This not only allows to reduce the number of necessary images from 9 to 4 , but also allows tiling the space with additional data to reconstruct additional high frequencies. This idea is illustrated in Fig. 13 To extend the first ring, we need only use 4 additional LR images to reconstruct the full ring as explained in Sec. III The only requirement is the ability to illuminate the sample with a higher frequency modulation, with saturated excitation for example [31]. The required number of images is then decreased to $8(4+4)$ instead of 21 with the classical SI approach.

The results of this experiment are simulated in Fig. 14

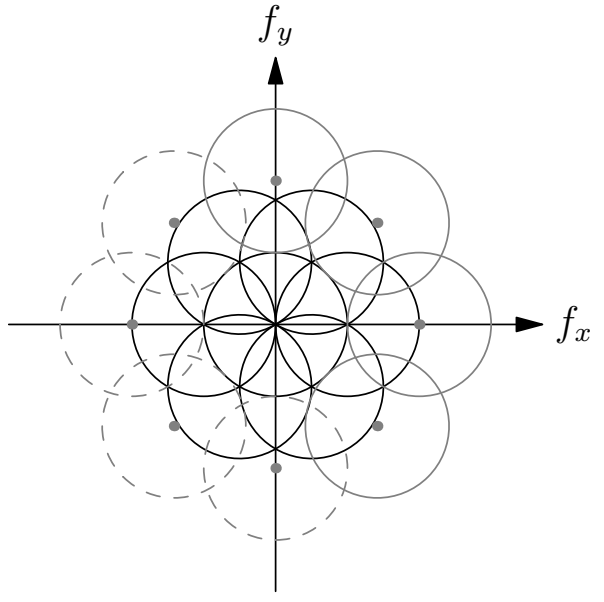

Fig. 13. Tiling for estimation of a second ring. The black pattern (first ring) is obtained with the illumination presented in Fig. 2. The gray pattern corresponds to the second ring. The gray dashed pattern is the conjugate of the gray pattern.

Figs. $14 \mathrm{~b}$ and $14 \mathrm{e}$ are the zoom and logarithm of the absolute value of DFT-2D of the "test pattern", with only the first ring. Figs. $14 \mathrm{c}$ and $14 \mathrm{f}$ are the results with the second ring, with 8 LR images. The $\mathrm{SNR} \approx 80 \mathrm{~dB}$ for both experiments. The gain is clearly visible, in direct and in Fourier domains. Numerical evaluation provides ISNR $\approx 8.65 \mathrm{~dB}$ and $\mathrm{SSIM} \approx 0.67$ for 8 LR images in comparison to ISNR $\approx 6.1 \mathrm{~dB}$ and SSIM $\approx 0.65$ for $4 \mathrm{LR}$ images.

\section{B. Real data}

We have tested our method on real fluorescence microscopy data. Fig. 16 is an example of image reconstruction through a SIM device built in our laboratory. The optical setup is based on a commercial microscope body (Olympus IX81). A schematic diagram of the system is presented in Fig. 15 Fig. $16 \mathrm{a}$ is one example of LR fluorescent images obtained with a standard wide field microscope [34]. The excitation laser light wavelength is $488 \mathrm{~nm}$. The excitation filter is a band pass $488 \pm 10 \mathrm{~nm}$ (Chroma Z488/10x) and the emmision filter is a long pass $500 \mathrm{~nm}$ (Chroma HQ500LP). The dichroic is a T495lpxr. Imaged sample in Fig. 16 are actin filaments labeled with quantum dots (QDs) and Figs. 17 and 18 are QDs is solution spread over a cover slip.

The standard illumination is replaced by a fringe projection setup to produce the amplitude modulation. Laser light was 


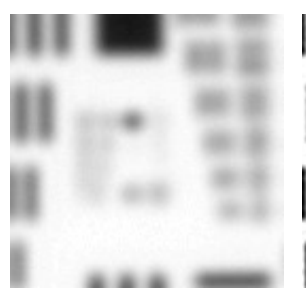

(a) Data

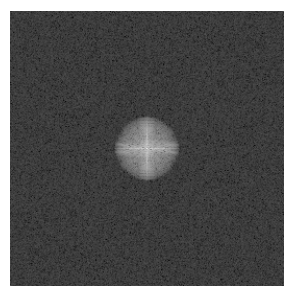

(d) Data

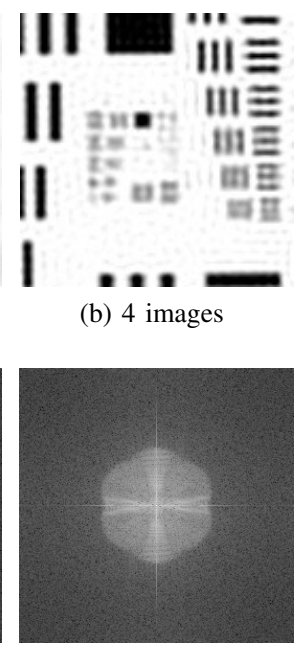

(e) 4 images

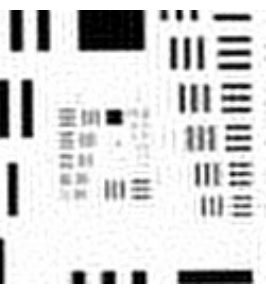

(c) 8 images

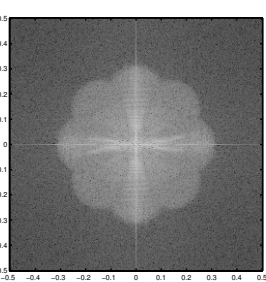

(f) 8 images
Fig. 14. Illustration of reconstruction improvement through additional high frequencies measurements. The images on the bottom row are the spectra of the images on the upper row (both axis in reduced frequency).

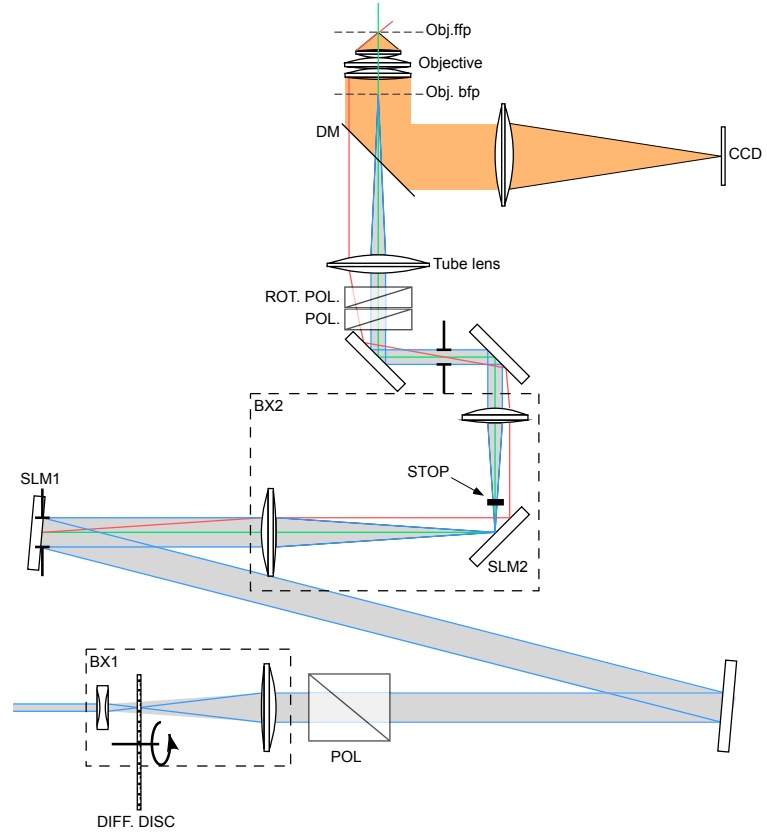

Fig. 15. The optical setup. BX1, BX2: beam expanders; DIFF.DISC: rotating diffusing disk; POL: polarizer (Glan-Taylor type); ROT. POL: liquid crystal polarization rotator; SLM1, SLM2: spatial light modulators (HoloEye phase-only 1080p); Tube lens: microscope illuminator tube lens; Obj. bfp: microscope objective back focal plane. DM: dichroic mirror (excitation and emission filters are not shown) ; obj: microscope objective. The incoherent fluorescent emission (in orange) is imaged on the CCD array. The diffusing disc is used to break the laser beam spatial coherence, and thus to restrict the localization of interference fringes inside the sample within the objective depth of field. The green ray represents the zeroth diffraction order chief ray and the red line is one of the $+/-1$ diffraction order chief ray. scrambled by a rotating diffuser to reduce spacial coherence (to have a better optical cut and reduce the influence of the out of focus light). The fringe pattern is generated by coherent illumination of a spatial light modulator (SLM) displaying a periodic structure [35]. We selected only the +1 and -1 diffraction orders by beam blocking the zero and superior orders. Both beams were focused in the back focal plane of the objective lens which made them interfere to form the illumination pattern with lateral sinusoidal structure and extending roughly $350 \mathrm{~nm}$ in the axial direction. Parameters of the illumination were estimated using cross-correlation techniques described in [12], [13]. A 16-bits camera was used with a $\times 100$ zoom and a pixel size of $8 \times 8 \mu \mathrm{m}$ as pixel size.

Fig. $16 \mathrm{~b}$ is the result of our reconstruction algorithm when using 9 LR images, 3 LR images in each orientation as described in Sec. [I-C The reconstruction Fig. 16c is the result obtained when using only 4 LR images extracted from the data set.

The results clearly show an improvement both in resolution and contrast. Filaments are more visible than in standard microscopy and small details are now visible. Structured illumination clearly improves spatial resolution. The results obtained with 9 and 4 images are very similar, demonstrating the capability of the proposed approach to effectively decrease the required number of LR images. The differences between Figs. $16 \mathrm{~b}$ and $16 \mathrm{c}$ are mostly due to contrast.

Figs. 17 and 18 demonstrate the effectiveness of our approach also when used on different types of samples, like these images of quantum dots (QDs) on a flat surface. Note that here we are not seeing individual QDs but rather aggregates. The differences between the classical SIM approach $17 \mathrm{~b}$ and our SIM method $17 \mathrm{c}$ are minimal. On Fig. 18 and Fig. 19 , the gain in resolution brought in by structured illumination can be appreciated by the fact that the QD aggregates that are not resolved by standard wide-field microscopy are well separated by SIM (Fig. 19c), irrespective of using 9 or just 4 images.

\section{CONClusion}

This paper has presented a novel approach for image reconstruction in Structured Illumination Microscopy. The proposed algorithm allows overcoming several drawbacks of existing approaches like automatic parameter estimation, reduction of necessary images and quantitative evaluation of the uncertainty. This is done through an inverse problem approach conducted within the Bayesian framework.

As illustrated in Sec. IV-B the reconstruction is very sensitive to the modulation parameters used in the algorithm which may constitute a serious difficulty in practice. Several works already exist to address this point [36]-[38]. One solution would be the joint estimation of these parameters together with the image and hyper-parameters, all within the same framework. This approach would lead to a myopic or semiblind image reconstruction [22].

Other perspectives are the use of different models for the noise, like Poisson, often encountered in microscopy. Different and more edge-preserving models for the image can also be considered like TV [39] or Huber-like [40] potentials. 


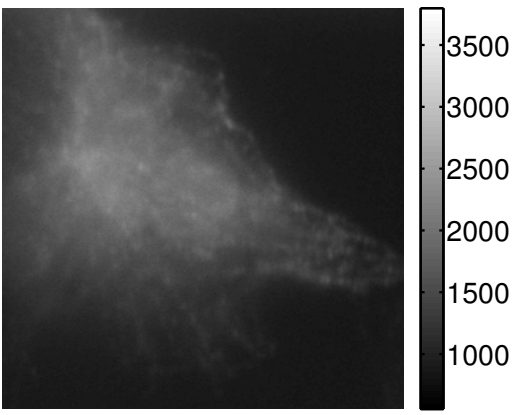

(a) WFM image

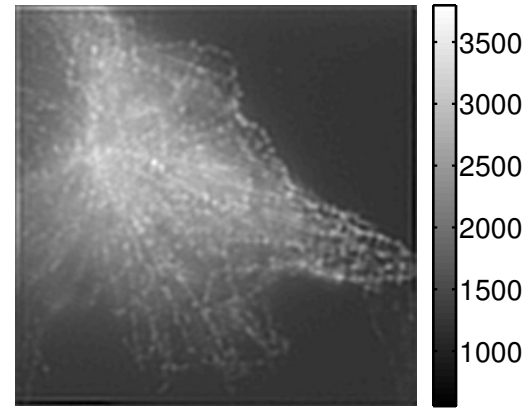

(b) SIM with 9 images

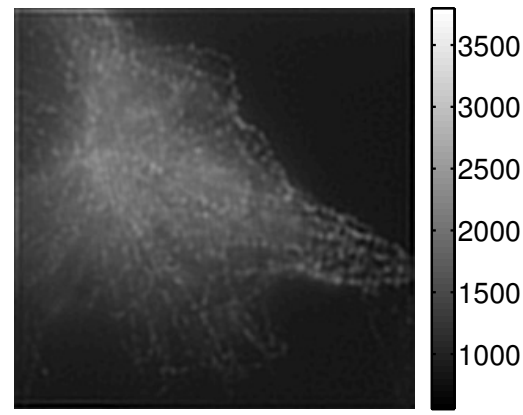

(c) SIM with 4 images

Fig. 16. Images of actin filaments labeled with QDs. (a) one example of LR image. (b) SIM reconstruction when 9 images are used. (c) SIM reconstruction when only 4 images are used. Field of view is $20 \times 20 \mu \mathrm{m}$.

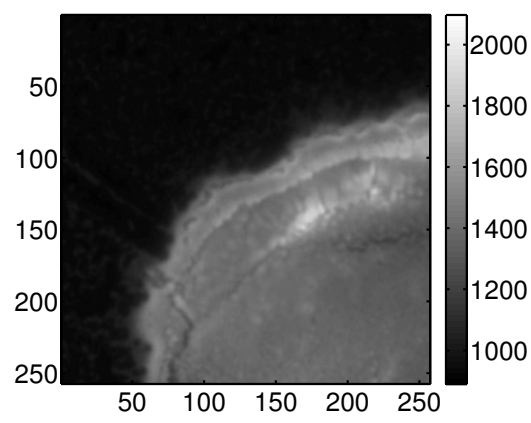

(a) WFM image

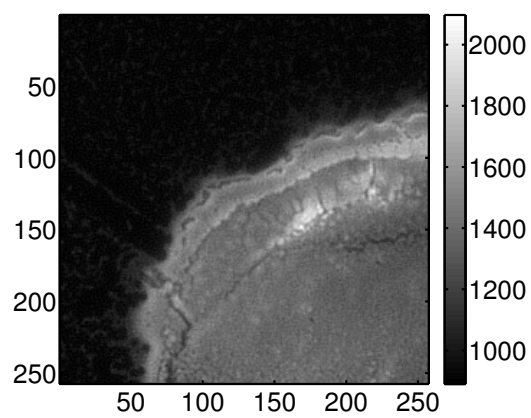

(b) SIM with 9 images

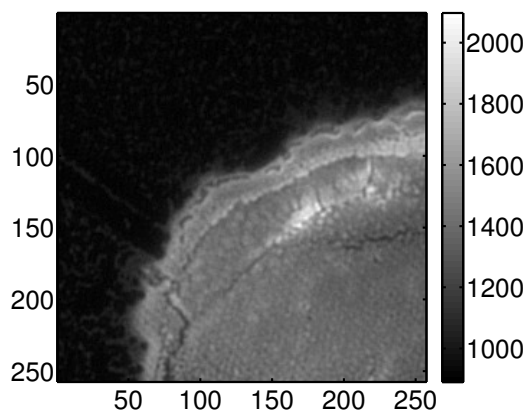

(c) SIM with 4 images

Fig. 17. Images of QDs dried on a glass surface and forming continuous aggregates. (a) widefield image. (b) SIM reconstruction with 9 images. (c) SIM reconstruction when only 4 images are used. Field of view is $20 \times 20 \mu \mathrm{m}$.

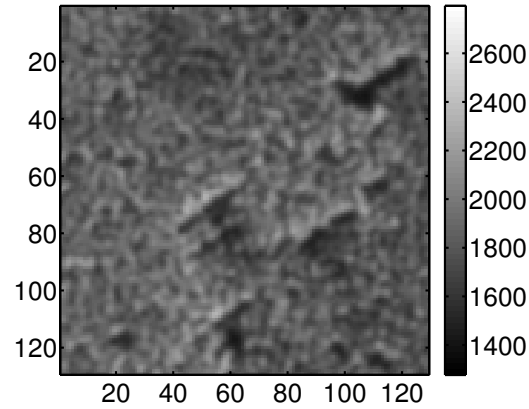

(a) WFM image

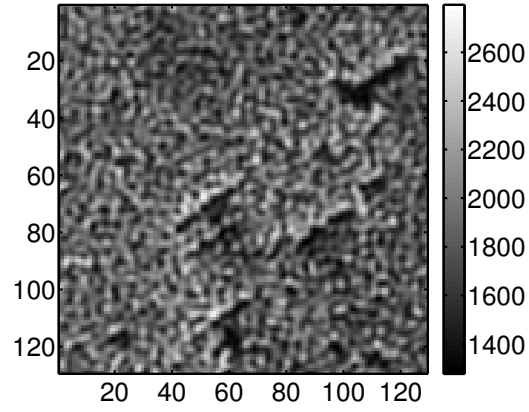

(b) SIM with 9 images

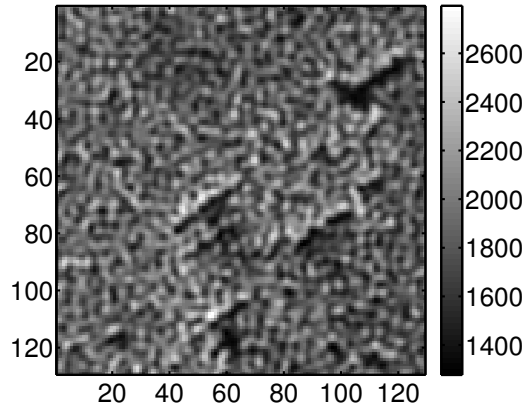

(c) SIM with 4 images

Fig. 18. Images of QDs dried on a glass surface and forming sparse aggregates. (a) widefield image. (b) SIM reconstruction with 9 images. (c) SIM reconstruction when only 4 images are used. Field of view is $10 \times 10 \mu \mathrm{m}$.

However, these kinds of models, because of the unknown normalisation constant of the law, do not allow a straightforward estimation of hyper-parameters.

\section{APPENDIX A}

SiMULATION OF CONDITIONAL POSTERIOR LAW OF THE IMAGE

The conditional posterior law of the image is Gaussian

$$
p\left(\boldsymbol{f} \mid, \gamma_{\boldsymbol{n}}{ }^{(k)}, \gamma_{\boldsymbol{f}}{ }^{(k)}, \boldsymbol{g}\right) \propto \mathcal{N}\left(\boldsymbol{m}_{\boldsymbol{f}}, \boldsymbol{\Sigma}_{\boldsymbol{f}}\right)
$$

where the mean and covariance matrix are

$$
\begin{aligned}
\boldsymbol{m}_{\boldsymbol{f}} & =\boldsymbol{\Sigma}_{\boldsymbol{f}}^{-1} \boldsymbol{R}^{t} \boldsymbol{M}^{t} \overline{\boldsymbol{H}}^{t} \boldsymbol{\Sigma}_{\boldsymbol{n}}^{-1} \boldsymbol{g}, \\
\boldsymbol{\Sigma}_{\boldsymbol{f}} & =\boldsymbol{R}^{t} \boldsymbol{M}^{t} \overline{\boldsymbol{H}}^{t} \boldsymbol{\Sigma}_{\boldsymbol{n}}^{-1} \overline{\boldsymbol{H}} \boldsymbol{M} \boldsymbol{R}+\gamma_{\boldsymbol{f}} \boldsymbol{D}^{t} \boldsymbol{D} .
\end{aligned}
$$

The difficulties are 1) the high dimension law (the number of pixels in HR image) and 2) the correlation present in $\boldsymbol{\Sigma}_{\boldsymbol{f}}$ that embed $\boldsymbol{H}$. The proposed solution is based on the same approach as in [29], [30] but the resolution is done with an iterative optimization algorithm instead of an explicit inversion of the matrix.

Proposition A.1: Consider a non-stationary Gaussian law 


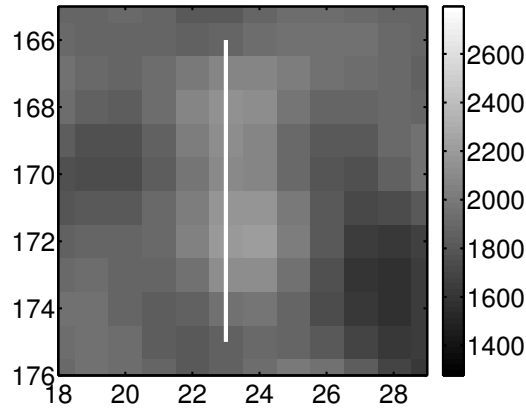

(a) Widefield

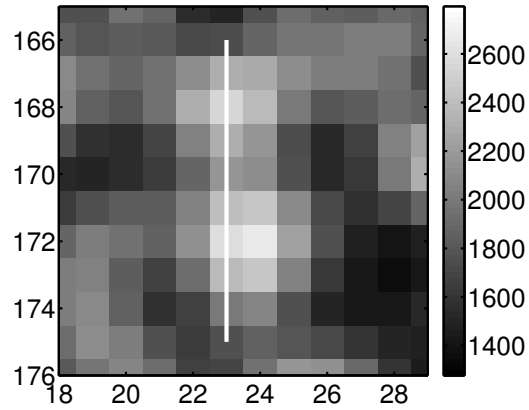

(b) SIM with 9

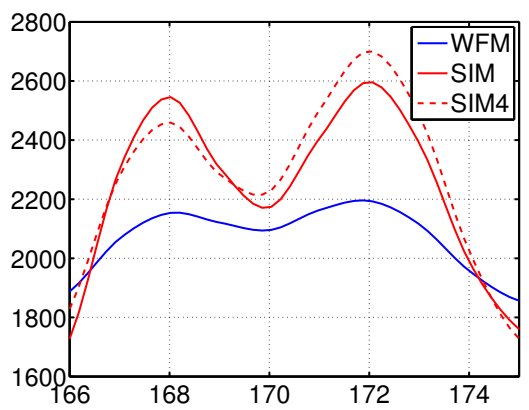

(c) Profiles

Fig. 19. Profile of a line in Fig. 18 (a) zoom on QD aggregates in the widefield image. (b) zoom on QD aggregates in the SIM reconstructed image with 9 LR images. (c) profile of the lines through the QD aggregates widefield image (WFM) and the SIM reconstruction with 9 and 4 images. The gain in resolution can be appreciated. Field of view is $960 \times 960 \mathrm{~nm}$.

$\mathcal{N}\left(\boldsymbol{m}_{f}, \boldsymbol{\Sigma}_{\boldsymbol{f}}\right)$, where

$$
\begin{aligned}
\boldsymbol{m}_{\boldsymbol{f}} & =\boldsymbol{\Sigma}_{\boldsymbol{f}}^{-1} \boldsymbol{A}^{t} \boldsymbol{\Sigma}_{n}^{-1} \boldsymbol{g} \\
\boldsymbol{\Sigma}_{\boldsymbol{f}} & =\boldsymbol{A}^{t} \boldsymbol{\Sigma}_{n}^{-1} \boldsymbol{A}+\boldsymbol{R}_{\boldsymbol{f}}^{-1} .
\end{aligned}
$$

If $\widetilde{\boldsymbol{g}}$ and $\widetilde{\boldsymbol{m}}$ are drawn by

$$
\widetilde{\boldsymbol{g}} \sim \mathcal{N}\left(\boldsymbol{g}, \boldsymbol{\Sigma}_{\boldsymbol{n}}\right) \quad \text { and } \quad \widetilde{\boldsymbol{m}} \sim \mathcal{N}\left(\mathbf{0}, \boldsymbol{R}_{\boldsymbol{f}}\right)
$$

which means that data and prior mean are perturbed with their respective prior law, then the minimum is defined by

$$
\begin{aligned}
\widehat{\boldsymbol{f}} & =\underset{\boldsymbol{f}}{\arg \min }(\widetilde{\boldsymbol{g}}-\boldsymbol{A} \boldsymbol{f})^{t} \boldsymbol{\Sigma}_{\boldsymbol{n}}^{-1}(\widetilde{\boldsymbol{g}}-\boldsymbol{A} \boldsymbol{f})+ \\
& (\boldsymbol{f}-\widetilde{\boldsymbol{m}})^{t} \boldsymbol{R}_{\boldsymbol{f}}^{-1}(\boldsymbol{f}-\tilde{\boldsymbol{m}}) \\
& =\boldsymbol{\Sigma}_{\boldsymbol{f}}^{-1}\left(\boldsymbol{A}^{t} \boldsymbol{\Sigma}_{\boldsymbol{n}}^{-1} \widetilde{\boldsymbol{g}}+\boldsymbol{R}_{\boldsymbol{f}} \widetilde{\boldsymbol{m}}\right)
\end{aligned}
$$

following a Gaussian law with mean $\boldsymbol{m}_{\boldsymbol{f}}$ and covariance $\boldsymbol{\Sigma}_{\boldsymbol{f}}$.

Proof: The variable $\widehat{f}$ is Gaussian as it is a linear combination of two Gaussian random variables. The mean and covariance are calculated below. From [47, we have

$$
\begin{aligned}
\mathbb{E}[\widehat{\boldsymbol{f}}] & =\boldsymbol{\Sigma}_{\boldsymbol{f}}^{-1} \mathbb{E}\left[\boldsymbol{A}^{t} \boldsymbol{\Sigma}_{\boldsymbol{n}}^{-1} \widetilde{\boldsymbol{g}}+\boldsymbol{R}_{\boldsymbol{f}}^{-1} \widetilde{\boldsymbol{m}}\right] \\
& =\boldsymbol{\Sigma}_{\boldsymbol{f}}^{-1}\left(\boldsymbol{A}^{t} \boldsymbol{\Sigma}_{\boldsymbol{n}}^{-1} \mathbb{E}[\widetilde{\boldsymbol{g}}]+\boldsymbol{R}_{\boldsymbol{f}}^{-1} \mathbb{E}[\widetilde{\boldsymbol{m}}]\right) \\
& =\boldsymbol{\Sigma}_{\boldsymbol{f}}^{-1} \boldsymbol{A}^{t} \boldsymbol{\Sigma}_{\boldsymbol{n}}^{-1} \boldsymbol{g} \\
& =\boldsymbol{m}_{\boldsymbol{f}} .
\end{aligned}
$$

From (47) and (48), we have

$$
\widehat{\boldsymbol{f}}-\mathbb{E}[\widehat{\boldsymbol{f}}]=\boldsymbol{\Sigma}_{\boldsymbol{f}}\left[\boldsymbol{A}^{t} \boldsymbol{\Sigma}_{\boldsymbol{n}}^{-1}(\widetilde{\boldsymbol{g}}-\boldsymbol{g})+\boldsymbol{R}_{\boldsymbol{f}}^{-1} \widetilde{\boldsymbol{m}}\right] .
$$

So, the covariance matrix is

$$
\begin{aligned}
& \mathbb{V}[\widehat{\boldsymbol{f}}]=\mathbb{E}\left[(\widehat{\boldsymbol{f}}-\mathbb{E}[\widehat{\boldsymbol{f}}])(\widehat{\boldsymbol{f}}-\mathbb{E}[\widehat{\boldsymbol{f}}])^{t}\right] \\
& =\boldsymbol{\Sigma}_{\boldsymbol{f}}\left[\boldsymbol{A}^{t} \boldsymbol{\Sigma}_{\boldsymbol{n}}^{-1} \mathbb{E}\left[(\widetilde{\boldsymbol{g}}-\boldsymbol{g})(\widetilde{\boldsymbol{g}}-\boldsymbol{g})^{t}\right] \boldsymbol{\Sigma}_{\boldsymbol{n}}^{-1} \boldsymbol{A}+\right. \\
& \left.\boldsymbol{R}_{\boldsymbol{f}}^{-1} \mathbb{E}\left[\widetilde{\boldsymbol{m}} \widetilde{\boldsymbol{m}}^{t}\right] \boldsymbol{R}_{\boldsymbol{f}}^{-1}\right] \boldsymbol{\Sigma}_{\boldsymbol{f}} \\
& =\boldsymbol{\Sigma}_{\boldsymbol{f}}\left[\boldsymbol{A}^{t} \boldsymbol{\Sigma}_{\boldsymbol{n}}^{-1} \mathrm{E}\left[\boldsymbol{\delta}_{\boldsymbol{g}} \boldsymbol{\delta}_{\boldsymbol{g}}^{t}\right] \boldsymbol{\Sigma}_{\boldsymbol{n}}^{-1} \boldsymbol{A}+\right. \\
& \left.\boldsymbol{R}_{f}^{-1} \mathbb{E}\left[\boldsymbol{\delta}_{f} \boldsymbol{\delta}_{f}^{t}\right] \boldsymbol{R}_{f}^{-1}\right] \boldsymbol{\Sigma}_{\boldsymbol{f}} \\
& =\boldsymbol{\Sigma}_{\boldsymbol{f}}\left[\boldsymbol{A}^{t} \boldsymbol{\Sigma}_{\boldsymbol{n}}^{-1} \boldsymbol{\Sigma}_{\boldsymbol{n}} \boldsymbol{\Sigma}_{\boldsymbol{n}}^{-1} \boldsymbol{A}+\boldsymbol{R}_{\boldsymbol{f}}^{-1} \boldsymbol{R}_{\boldsymbol{f}} \boldsymbol{R}_{\boldsymbol{f}}^{-1}\right] \boldsymbol{\Sigma}_{\boldsymbol{f}}
\end{aligned}
$$

$$
\begin{aligned}
& =\boldsymbol{\Sigma}_{\boldsymbol{f}}\left[\boldsymbol{A}^{t} \boldsymbol{\Sigma}_{n}^{-1} \boldsymbol{A}+\boldsymbol{R}_{\boldsymbol{f}}^{-1}\right] \boldsymbol{\Sigma}_{\boldsymbol{f}}=\boldsymbol{\Sigma}_{\boldsymbol{f}} \boldsymbol{\Sigma}_{\boldsymbol{f}}^{-1} \boldsymbol{\Sigma}_{\boldsymbol{f}} \\
& =\boldsymbol{\Sigma}_{\boldsymbol{f}} .
\end{aligned}
$$

Then $\widehat{f}$ follows the target law $\mathcal{N}\left(\boldsymbol{m}_{\boldsymbol{f}}, \boldsymbol{\Sigma}_{\boldsymbol{f}}\right)$.

\section{REFERENCES}

[1] M. G. L. Gustafsson, "Surpassing the lateral resolution limit by a factor of two using structured illumination microscopy," Journal of Microscopy, vol. 198, pp. 82-87, May 2000.

[2] V. Galy, J.-C. Olivo-Marin, H. Scherthan, V. Doye, N. Rascalou, and U. Nehrbass, "Nuclear pore complexes in the organization of silent telomeric chromatin," Nature, vol. 403, no. 6765, pp. 108-12, 2000.

[3] N. Arhel, A. Genovesio, K. Kim, S. Miko, E. Perret, J. Olivo-Marin, S. Shorte, and P. Charneau, "Quantitative four-dimensional tracking of cytoplasmic and nuclear hiv-1 complexes," Nature Methods, vol. 3, no. 10 , pp. 817-824, 2006.

[4] C. Vonesch, F. Aguet, J.-L. Vonesch, and M. Unser, "The colored revolution of bioimaging," IEEE Signal Processing Magazine, pp. 2031, May 2006.

[5] S. W. Hell, "Microscopy and its focal switch," Nature methods, vol. 6, no. 1, pp. 24-32, January 2009.

[6] R. Betzig, "Near-field Scanning Optical Microscopy," in Lasers and Electro-Optics Society Annual Meeting, 1992. LEOS'92, Conference Proceedings. IEEE, 2002, p. 324.

[7] B. Huang, W. Wang, M. Bates, and X. Zhuang, "Three-dimensional super-resolution imaging by stochastic optical reconstruction microscopy," Science, vol. 319, no. 5864, pp. 810-3, 2008.

[8] G. Brakenhoff, H. van der Voort, M. Baarslag, B. Mans, J. Oud, R. Zwart, and R. van Driel, "Visualization and analysis techniques for three dimensional information acquired by confocal microscopy," Scanning microscopy, vol. 2, no. 4, pp. 1831-8, 1988.

[9] W. Denk, J. Strickler, and W. Webb, "Two-photon laser scanning fluorescence microscopy," Science, vol. 248, no. 4951, pp. 73-6, 1990.

[10] S. Hell, K. Bahlmann, M. Schrader, A. Soini, H. Malak, I. Gryczynski, and J. Lakowicz, "Three-photon excitation in fluorescence microscopy," J. Biomed. Opt, vol. 1, no. 1, pp. 71-74, 1996.

[11] J. W. Goodman, Introduction to Fourier Optics, 2nd ed. McGraw-Hill International Editions, 1996.

[12] M. G. L. Gustafsson, L. Shao, P. M. Carlton, C. J. R. Wang, I. N. Golubovskaya, W. Z. Cande, D. A. Agard, and J. W. Sedat, "Threedimensional resolution doubling in wide-field fluorescence microscopy by structured illumination," Biophysical Journal, vol. 94, no. 12, pp. 4957-4970, June 2008.

[13] O. Mandula, "Patterned excitation microsopy," Master's thesis, King's College London, 2008.

[14] J. Conchello, "Image estimation for structured-illumination microscopy," in Proceedings of SPIE, vol. 5701, 2005, p. 34.

[15] G. Demoment, "Image reconstruction and restoration: Overview of common estimation structure and problems," IEEE Trans. Acoust. Speech, Signal Processing, vol. ASSP-37, no. 12, pp. 2024-2036, December 1989.

[16] J. Idier, Ed., Bayesian Approach to Inverse Problems. London: ISTE Ltd and John Wiley \& Sons Inc., 2008. 
[17] H. Takeda, P. Milanfar, M. Protter, and M. Elad, "Super-Resolution without explicit subpixel motion estimation," IEEE Trans. Image Processing, vol. 18, no. 9, pp. 1958-1975, 2009.

[18] S. Farsiu, M. Robinson, M. Elad, and P. Milanfar, "Fast and robust multiframe super resolution," Image Processing, IEEE Transactions on, vol. 13, no. 10, pp. 1327-1344, October 2004.

[19] G. Demoment, Inverse problems: theory and applications. Lorient, France: Spring-Rolls, 1997.

[20] B. Zhang, J. M. Fadili, and J.-L. Starck, "Wavelets, ridgelets and curvelets for poisson noise removal," IEEE Trans. Image Processing, vol. 17, no. 7, pp. 1093-1108, 2008.

[21] M. Calder and R. A. Davis, "Introduction to Whittle (1953) 'The analysis of multiple stationary time series'," Breakthroughs in Statistics, vol. 3, pp. 141-148, 1997

[22] F. Orieux, J.-F. Giovannelli, and T. Rodet, "Bayesian estimation of regularization and PSF parameters for Wiener-Hunt deconvolution," $J$. Opt. Soc. Amer. (A), 2010.

[23] R. Molina, J. Mateos, and A. K. Katsaggelos, "Blind deconvolution using a variational approach to parameter, image, and blur estimation," IEEE Trans. Image Processing, vol. 15, no. 12, pp. 3715-3727, December 2006.

[24] C. Robert, The Bayesian Choice. Springer, 2007.

[25] R. E. Kass and L. Wasserman, "The selection of prior distributions by formal rules," J. Amer. Statist. Assoc., vol. 91, pp. 1343-1370, 1996.

[26] G. Winkler, Image Analysis, Random Fields and Markov Chain Monte Carlo Methods. Springer Verlag, Berlin, Germany, 2003.

[27] P. Brémaud, Markov Chains. Gibbs fields, Monte Carlo Simulation, and Queues, ser. Texts in Applied Mathematics 31. New York, NY: Spinger, 1999.

[28] S. Geman and D. Geman, "Stochastic relaxation, Gibbs distributions, and the Bayesian restoration of images," IEEE Trans. Pattern Anal. Mach. Intell., vol. 6, no. 6, pp. 721-741, November 1984

[29] H. Rue, "Fast sampling of Gaussian Markov random fields," J. R. Statist. Soc $B$, vol. 63, no. 2, pp. 325-338, 2001, échantillonnage par résolution d'un système linéaire.

[30] P. Lalanne, D. Prévost, and P. Chavel, "Stochastic artificial retinas: algorithm, optoelectronic circuits, and implementation," Appl. Opt, vol. 40, pp. 3861-3876, 2001

[31] R. Heintzmann, "Saturated patterned excitation microscopy with twodimensional excitation patterns," Micron, vol. 34, no. 6-7, pp. 283-291, 2003.

[32] J. Nocedal and S. J. Wright, Numerical Optimization, ser. Series in Operations Research. New York: Springer Verlag, 2000.

[33] Z. Wang and A. C. Bovik, "Mean square error : Love it or leave it ? A new look at signal fidelity measures." Signal Processing Magazine, IEEE, 2009

[34] P. F. G. Rodríguez, E. Sepulveda, B. Dubertret, and V. Loriette, "Axial coding in full-field microscopy using three-dimensional structured illumination implemented with no moving parts," Optics Letters, vol. 33, no. 14 , July 2008

[35] J. Rosen and Takeda, "Longitudinal spatial coherence applied for surface profilometry," Appl. Opt, pp. 4107-4111, 2000.

[36] L. Schaefer, D. Schuster, and J. Schaffer, "Structured illumination microscopy: artefact analysis and reduction utilizing a parameter optimization approach," J. of Microscopy, no. 216, pp. 165-174, 2004.

[37] S. Shroff, J. Fienup, and D. Williams, "Lateral superresolution using a posteriori phase shift estimation for a moving object: experimental results," JOSA A, vol. 27, no. 8, pp. 1770-1782, 2010.

[38] _ , "Phase-shift estimation in sinusoidally illuminated images for lateral superresolution," Journal of the Optical Society of America A, vol. 26, no. 2, pp. 413-424, 2009.

[39] G. Chantas, N. P. Galatsanos, R. Molina, and A. K. Katsaggelos, "Variational Bayesian image restoration with a product of spatially weighted total variation image priors," Image Processing, IEEE Transactions on, vol. 19, no. 2, pp. $351-362$, February 2010.

[40] P. J. Huber, Robust Statistics. New York, NY: John Wiley, 1981.

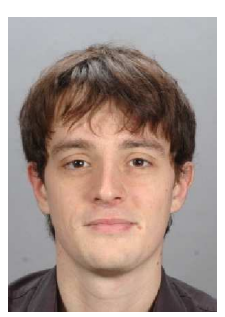

François Orieux received the $\mathrm{Ph}$. D. degree in signal processing from the Université Paris-Sud in 2009. He is currently at the Pasteur institute with the Quantitative Image Analysis unit. His research interests are statistical signal and image processing, inverse problems, Bayesian analysis and application to instrumentation problems.

Eduardo Sepulveda received the Electrical Engineer degree in 1989 from the University of Chile, Santiago and the Ph.D. degree in Computer Science in 1994 from the Universite Pierre et Marie Curie, Paris. He has been at LPEM since 2003, dedicated to develop patterned excitation microscopes. His research interest include image analysis, signal processing and optics.

Vincent Loriette received its $\mathrm{PhD}$ in astrophysics from the Université Denis Diderot in 1995. His research activities are split between the Laboratoire de l'accélérateur linéaire at the Université Pars Sud where he contributes to the development of the Virgo gravitational wave detector, and the Laboratoire de Physique et d'Etude des Matériaux where he builds original biological microscopes with improved performances and new capabilities.

Benoit Dubertret is a CNRS researcher at the Laboratoire de Physique et d'Etude des Matériaux located at the ESPCI in Paris, France. He received his $\mathrm{PhD}$ in 1998 at the University Louis Pasteur (Strasbourg, France). He did a post-doc in the laboratory of Albert Libchaber at the Rockefeller University (NY, USA). His research interests include semiconductor nanoparticle synthesis, characterisation and applications.

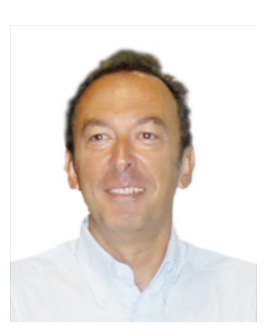

Jean-Cristophe Olivo-Marin is the head of the Quantitative Image Analysis Unit and the Chair of the Cell Biology and Infection Department at the Institut Pasteur, Paris. He holds a Ph.D. in Optics and Signal Processing and the "Habilitation à Diriger des Recherches" from the Institut d'Optique Théorique et Appliquée, University of Paris-Orsay. He was a co-founder of the Institut Pasteur Korea, Seoul, where he held a joint appointment as Chief Technology Officer from 2004 to 2005. Previous to that, he was a staff scientist from 1990 to 1998 at the European Molecular Biology Laboratory, Heidelberg. He is a specialist of image analysis of multidimensional microscopy images, computer vision and motion analysis for cellular dynamics. He is a Senior Member of IEEE, a member of SPS and EMBS, Chair of the Bio Imaging and Signal Processing Technical Committee (BISP-TC), the Pattern Recognition Society and member of the Editorial Board of the journal Medical Image Analysis. He has organized several special sessions dedicated to biological imaging at international biomedical conferences (ELMI'02, ELSO'03, ISBI'04, ICASSP'06, SPIE Wavelets'09) and was General Chair of the IEEE International Symposium on Biomedical Imaging held in Paris in 2008. 